\title{
Effects of Different Jumping Programs on Hip and Spine Bone Mineral Density in Pre-Menopausal Women
}

\author{
J. Eric Strong \\ Brigham Young University - Provo
}

Follow this and additional works at: https://scholarsarchive.byu.edu/etd

Part of the Exercise Science Commons

\section{BYU ScholarsArchive Citation}

Strong, J. Eric, "Effects of Different Jumping Programs on Hip and Spine Bone Mineral Density in PreMenopausal Women" (2004). Theses and Dissertations. 667.

https://scholarsarchive.byu.edu/etd/667

This Dissertation is brought to you for free and open access by BYU ScholarsArchive. It has been accepted for inclusion in Theses and Dissertations by an authorized administrator of BYU ScholarsArchive. For more information, please contact scholarsarchive@byu.edu, ellen_amatangelo@byu.edu. 
EFFECTS OF DIFFERENT JUMPING PROGRAMS ON HIP AND SPINE

BONE MINERAL DENSITY IN PRE-MENOPAUSAL WOMEN

by

John Eric Strong

A dissertation submitted to the faculty of

Brigham Young University

in partial fulfillment of the requirements for the degree of

Doctor of Philosophy

Department of Exercise Sciences

Brigham Young University

December 2004 


\title{
BRIGHAM YOUNG UNIVERSITY
}

\section{GRADUATE COMMITTEE APPROVAL}

\author{
of a dissertation submitted by \\ John Eric Strong
}

This dissertation has been read by each member of the following graduate committee and by majority vote has been found to be satisfactory.

Date

Date

Date

Date

Date
Larry A. Tucker, Chair

Brent Feland

Ronald L. Hager

Iain Hunter

Ray M. Merrill 


\section{BRIGHAM YOUNG UNIVERSITY}

As chair of the candidate's graduate committee, I have read the dissertation of John Eric Strong in its final form and have found that (1) its format, citations, and bibliographical style are consistent and acceptable and fulfill university and department style requirements; (2) its illustrative materials including figures, tables, and charts are in place; and (3) the final manuscript is satisfactory to the graduate committee and is ready for submission to the university library.

Date

Accepted for the Department
Larry A. Tucker

Chair, Graduate Committee
Ruel Barker

Chair, Department of Exercise Sciences

Accepted for the College

Gordon B. Lindsay

Associate Dean, College of Health and Human Performance 


\begin{abstract}
EFFECTS OF DIFFERENT JUMPING PROGRAMS ON HIP AND SPINE BONE MINERAL DENSITY IN PREMENOPAUSAL WOMEN

\author{
John Eric Strong \\ Department of Exercise Sciences \\ Doctor of Philosophy
}

\begin{abstract}
Sixty premenopausal women (age 25-50) finished a 16 week randomized controlled trial looking at the effects of different jump programs on hip and spine bone mineral density (BMD). Subjects were randomly assigned to a control group or one of two jumping groups. The Jump 10 group performed 10 jumps with 30 seconds of rest between jumps, twice daily, while the Jump 20 group performed the same protocol but with 20 jumps. At 8 weeks, BMD percent change $(\% \Delta)$ at the hip was significantly different among groups when age, weight change, and subject compliance were simultaneously adjusted. In particular, the Jump 20 group had a greater $\% \Delta$ in hip BMD compared to controls. However, $\% \Delta$ in BMD for the neck of the hip (NOH), trochanter, or spine was not different among groups at 8 weeks. Following 16 weeks of jumping, BMD $\% \Delta$ at the total hip and trochanter were significantly different among groups after adjusting for all covariates. Specifically, at the hip and trochanter, the Jump 10 and Jump 20 groups showed significantly greater $\% \Delta$ in BMD compared to controls. There were
\end{abstract}


no significant differences among groups for the BMD $\% \Delta$ at $\mathrm{NOH}$ or spine at 16 weeks. Within-group analyses revealed no significant changes from baseline to 16 weeks for the jumping groups, but controls lost significant BMD. In conclusion, after 16 weeks of high-impact jump training, BMD at the hip and trochanter can be improved by jumping 10 or 20 times, twice daily, with 30 seconds of rest between each jump, compared to controls. 


\section{ACKNOWLEDGMENTS}

I am so appreciative to Dr. Tucker for his guidance, mentoring, and influence on my doctoral program, research philosophy, and my project. I would also like to thank my committee for their support and guidance during my doctoral program. To my children, Casey and Emily, thanks for being understanding when school took me away from you. Most of all, I thank my wife Julie for constant support and encouragement from the beginning. I would never have achieved this without your love and support. 


\section{Table of Contents}

List of Tables..............................................................viii

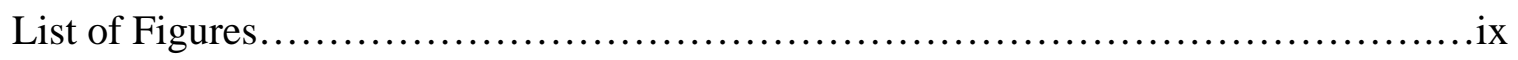

Effects of Different Jumping Programs on Hip and Spine Bone Mineral Density in

Premenopausal Women

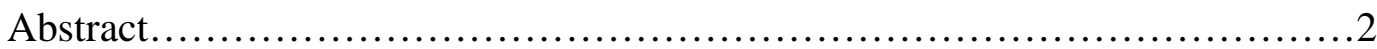

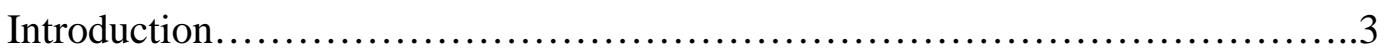

Materials and Methods.................................................6

Results...............................................................

Discussion.............................................................

References............................................................22

Appendix A Prospectus....................................................... 31

Introduction....................................................... 32

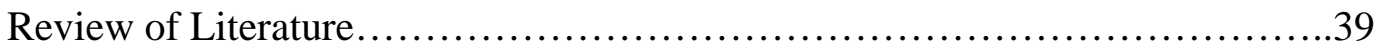

Methods...............................................................66

References.........................................................75

Appendix A-1 Informed Consent.......................................89

Appendix A-2 Screening Form..........................................94

Appendix A-3 Menstrual Cycle Questionnaire.............................96

Appendix A-4 Exercise Logs..........................................99 
List of Tables

Table

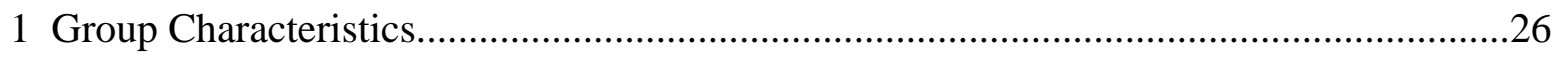

2 Comparison of Average Group Percent Change in Bone Mineral Density Over 8

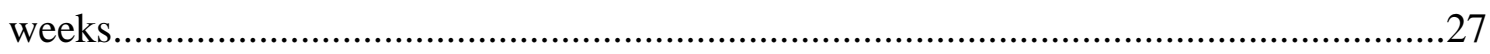

3 Comparison of Average Group Percent Change in Bone Mineral Density Over 16

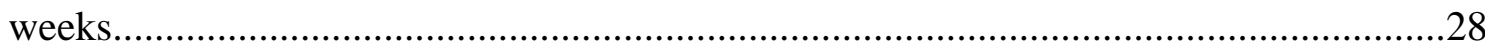




\section{List of Figures}

\section{Figure}

1. Mean Percent Change in Hip BMD Over 16 Weeks ...........................................29

2. Mean Percent Change in Trochanter BMD Over 16 Weeks.....................................30 
Effects of Different Jumping Programs on Hip and Spine Bone Mineral Density in Premenopausal Women

\author{
J. Eric Strong ${ }^{1}$ \\ Larry A. Tucker ${ }^{1}$ \\ Iain Hunter ${ }^{1}$ \\ Brent Feland ${ }^{1}$ \\ Ron Hager ${ }^{1}$ \\ Ray Merrill ${ }^{2}$
}

\footnotetext{
${ }^{1}$ Department of Exercise Sciences, Brigham Young University, Provo, UT 84602

${ }^{2}$ Department of Health Science, Brigham Young University, Provo, UT 84602
}

Running Title: Effects of Jumping on Bone Mineral Density in Women

Address all correspondence to J. Eric Strong

J. Eric Strong, PhD, PT

Department of Physical Therapy

Husson College

One College Circle

Bangor, ME 04401

Phone: (207) 973-1031

Email: strongj@husson.edu 
2 Effects of Jumping on Bone Mineral Density in Women

\section{ABSTRACT}

Sixty premenopausal women (age 25-50) finished a 16 week randomized controlled trial looking at the effects of different jump programs on hip and spine bone mineral density (BMD). Subjects were randomly assigned to a control group or one of two jumping groups. The Jump 10 group performed 10 jumps with 30 seconds of rest between jumps, twice daily, while the Jump 20 group performed the same protocol but with 20 jumps. At 8 weeks, BMD percent change $(\% \Delta)$ at the hip was significantly different among groups when age, weight change, and subject compliance were simultaneously adjusted. In particular, the Jump 20 group had a greater $\% \Delta$ in hip BMD compared to controls. However, $\% \Delta$ in BMD for the neck of the hip (NOH), trochanter, or spine was not different among groups at 8 weeks. Following 16 weeks of jumping, $\mathrm{BMD} \% \Delta$ at the total hip and trochanter were significantly different among groups after adjusting for all covariates. Specifically, at the hip and trochanter, the Jump 10 and Jump 20 groups showed significantly greater $\% \Delta$ in BMD compared to controls. There were no significant differences among groups for the $\mathrm{BMD} \% \Delta$ at $\mathrm{NOH}$ or spine at 16 weeks. Within-group analyses revealed no significant changes from baseline to 16 weeks for the jumping groups, but controls lost significant BMD. In conclusion, after 16 weeks of high-impact jump training, BMD at the hip and trochanter can be improved by jumping 10 or 20 times, twice daily, with 30 seconds of rest between each jump, compared to controls.

High-impact; exercise; bone mass 


\section{INTRODUCTION}

Peak bone mass or density is a major factor in determining the strength of bone. Research has found that bone mineral density accounts for $70 \%$ of total bone strength (1). Decreased bone density, as a result of age, will increase the risk of fracture. When bone loss is accelerated or if there is a suboptimal peak bone mineral density (BMD), osteoporosis can develop (15).

Osteoporosis is a disease characterized by increased fracture risk due to decreased bone content. The most common fracture sites associated with osteoporosis are the hip and lumbar vertebrae (spine). Each year there are approximately 300,000 and 700,000 hip and spine fractures, respectively, within the United States (2). By the year 2040, it is estimated that the number of hip fractures could triple in the United States (2).

Pain and disability are associated with any osteoporotic fracture. Hip fractures however, have been associated with an increase in the risk of mortality. It has been reported that one in four women who suffer a hip fracture will die within a year of suffering the fracture (2). The projected increase in hip fracture incidence and potential mortality may be lessened by increasing BMD before the onset of menopause, which accelerates BMD loss (17).

Prevention of osteoporosis is mostly a function of addressing the modifiable risk factors that a woman possesses. Physical activity, a modifiable risk factor, has been shown to provide a stimulus to bone that increases mineralization in areas of new stress (14). The stress from physical activity must be in a manner to which bone is not accustomed in order to promote bone strengthening. Activities of daily living in 


\section{Effects of Jumping on Bone Mineral Density in Women}

nonsedentary individuals are not capable of increasing BMD, because the stresses do not reach the osteogenic strain threshold (28). Exercise, a subset of physical activity, may be capable of generating stresses that cross the osteogenic strain threshold.

Exercise can be divided into impact and nonnimpact activities based on the loading pattern. Both types of exercise have muscle contractions producing strains on bone, but ground reaction forces (GRF) are only involved with impact exercise. Nonimpact exercise, such as biking or swimming, have not been effective in changing BMD at the hip $(7,9,13)$ nor spine $(7,13)$ in premenopausal women. However, impact activities, like running $(5,9,10)$, weight lifting $(10,13)$, and certain sporting events such as basketball $(7,13,18)$, volleyball $(7,18)$, or gymnastics $(18)$, have increased or are associated with increases in hip BMD in premenopausal women due to the large GRF.

Jumping is also an impact exercise and several research projects have studied its effect on hip and spine BMD in premenopausal women. These studies have focused specifically on the magnitude of $\operatorname{GRF}(3,4,12)$, the number of repetitions performed $(3$, $4,12,25)$, and the number of times a session was performed $(3,4,12,25)$.

In jumping studies with premenopausal women, GRF have been reported to be between 2.0 and 6.0 times body weight $(3,4,12)$. Jumping repetitions have varied among studies with some subjects jumping rope for a certain amount of time or performing between 33 and 100 jumps in one jumping session $(3,4,12,25)$. The number of sessions that subjects performed each week have varied from 3 to 7 times per week (3, $4,12,25)$. Results of these studies have been positive for hip BMD, but mixed for spine BMD for premenopausal women $(3,4,12,25)$. 
Research on BMD and bone loading in rodents has also focused on the number of repetitions $(18,20,27-29)$, along with the frequency of daily loading sessions (27-29), and rest between each loading repetition $(27,33)$. Results from these studies have reported a loss in bone sensitivity to the loading stimulus after the first couple of loading repetitions $(26,27)$. If a loading stimulus occurs before the bone restores sensitivity, the bone may have a diminished response or no response at all $(22,24)$. Research in rodents has also determined that shorter exercise sessions of high magnitude strain followed by rest between repetitions and sets may be more beneficial than one long exercise session each day (21-24). These findings from rodent studies raise the question of whether or not a delay between jumps and/or sets might promote increased BMD in premenopausal women.

The purpose of this research project was to determine the effects of two different jumping protocols on percent change in BMD at the hip and spine compared to a control group. Unlike previous jumping studies in premenopausal women, these jumping protocols used fewer jump repetitions, divided the jump repetitions into two daily sessions, included 30 seconds of rest between jump repetitions, along with at least 8 hours of rest between each jump session. All assigned activities were completed in the subjects' homes. Secondary objectives were to determine if percent changes in hip and spine BMD were independent of age, changes in body weight, calcium intake, ground reaction force, and activity compliance within the sample. 
6 Effects of Jumping on Bone Mineral Density in Women

\section{MATERIALS AND METHODS}

Design

The study used a pretest-midtest-posttest randomized-groups design with two treatment groups and one control group. The midtest and posttest occurred at 8 and 16 weeks, respectively. The study was approved by the university Internal Review Board. Subjects

Premenopausal women between 25-50 years old were recruited for the study through newspaper advertisements, flyers, referrals, and phone calls. Each potential subject was screened for possible exclusion from the study with a short survey. Subjects were excluded if they had been diagnosed in the last 12 months with a surgical or medical condition that would affect BMD (thyroid or parathyroid disorder, renal or hepatic dysfunction, digestive disorder) (20) or affect exercise compliance (arthritis, back pain, heart condition, or malignancy). Exclusions were also made of individuals who had not maintained their exercise levels for at least six months, had lost or gained greater than 10 pounds within the last six months, were current dieters, smoked, were less than 25 or greater than 50 years of age, had a hip BMD $t$-score $>1.0$ or $<-2.0$. Irregular (varying cycle length) or infrequent menstrual cycles (less than 10 cycles per year) (7), a hysterectomy without hormone replacement, or a hysterectomy with hormone replacement within the last six months also led to exclusion. Users of glucocorticoids, anticonvulsants, or thiazide diuretic medications (20) within the last six months were excluded, along with women who were pregnant, had breastfed within the previous six 
months, or had delivered a baby within the previous 12 months. A total of 88 qualified subjects were randomly assigned to one of two treatment groups or a control group. Experimental Protocol

Qualified subjects were scheduled for an orientation meeting that corresponded to group assignment. Subjects read and signed an informed consent as well as filled out a menstrual cycle questionnaire. Subjects were requested to maintain their current lifestyle with respect to eating habits, supplements, and exercise, for the duration of the study. A Block 98 food frequency questionnaire (Berkeley Nutritional Services, Berkeley, CA) (6, $16,19)$ was mailed to each subject, which was returned at the eight-week appointment. Subjects returned all questionnaires.

Data were collected at baseline, 4, 8, 12, and 16 weeks. At baseline, 8, and 16 weeks, subjects were weighed, had hip and spine BMD scans, and jumped 10 times on a force plate with 30 seconds separating each jump. Subjects performed the jumps using the same jumping protocol as performed at home. At weeks 4 and 12, subjects performed their assigned activities in front of a research assistant who corrected any deviations from the form described below. Also, subjects were weighed on an electronic scale.

An exercise log was provided at each appointment to assess compliance to the jumping activities described below. Completed logs were returned at the subjects' next appointment. Compliance was determined from the logs and calculated as the percent of total activity performed. The first month was excluded from compliance scores due to the transitional nature of the jump protocols for that month. Subjects also called weekly 


\section{Effects of Jumping on Bone Mineral Density in Women}

and left a message about their activity. Subjects who did not report in received a phone call to check on their activity.

Treatment Group Protocols. Subjects in the treatment groups participated in one of two jumping programs, whereas control subjects participated in a stretching program. Stretching has not been reported to promote osteogenic activity (11). Engaging in a regular stretching program was designed to foster the perception of full participation in the study, in order to decrease attrition from the study. All study-related exercise was completed in the subjects' homes.

For subjects in the treatment groups, jumps were performed without shoes and socks. Subjects jumped vertically as high as possible from a hard surface covered with a thin piece of carpet provided by the researchers. The carpet used was the same for all subjects and measured $0.5 \mathrm{~cm}$ thick. Subjects were taught to bend slightly at the hips, knees, and ankles and use their arms for countermovement, helping increase jump height. Subjects were instructed to land on the balls of their feet, followed by a heel strike along with a slight bend at the hips, knees, and ankles. After landing, subjects rested for 30 seconds between jumps. Jumping sessions were performed six days per week according to the protocol of the jump group.

A progression of jump repetitions was included in order to condition the subjects' body to the impact of treatment. Initially, subjects in the treatment groups jumped from the floor five times for each session that was to be performed during the first week. During the second week, jumps from the floor increased to 10 for each session for all treatment groups. By the second week, one treatment group was performing their total 
number of repetitions (10 repetitions). Beginning with the third week, the other treatment group performed 15 jumps for each session, which was increased to 20 jumps in each session by the fourth week. By the beginning of the fourth week, all groups were performing the maximal number of jumps as set forth in the following protocols:

Jump10. Subjects stood on a mat-covered hard surface and jumped as high as possible. After landing, the subjects rested for 30 seconds before performing the next jump. Subjects completed 10 jumps at each session with at least eight hours separating the two daily sessions. The full protocol began at the start of the second week. After randomization, a total of 29 women were assigned to this group.

Jump 20. Subjects stood on a mat-covered hard surface and jumped as high as possible. After landing, the subjects rested for 30 seconds before performing the next jump. Subjects completed 20 jumps at each session with at least eight hours separating the two daily sessions. The full protocol began at the start of the fourth week. After randomization, a total of 29 women were assigned to this group.

Control Group. Subjects performed three thigh stretches for a total of ten minutes per day, six days per week. Subjects were instructed to stretch their hamstrings, gluteals, and hip adductors until mild discomfort occurred within each muscle. Subjects then held this stretch for 30 seconds before switching to stretch the opposite leg. After randomization, a total of 30 women were assigned to this group.

The following stretches were performed. For hamstrings, the subject sat on the ground with one leg straight and the other bent at the knee to allow the sole of the foot to touch the inside of the opposite thigh. For gluteals, each subject sat in a chair with one 
10 Effects of Jumping on Bone Mineral Density in Women

leg crossed over the other. The subject leaned her trunk forward until a mild discomfort was felt in the hip. Finally, the hip adductors were stretched by standing with the feet wide apart and the subject bending one knee and leaning to that side. The opposite leg remained straight.

\section{Instrumentation and Measurement Methods}

Anthropometric Measures. Height was measured to the nearest 0.001 meter using a wall-mounted stadiometer, model 439 (Cardinal Scale Manufacturing, Webb City, MO). Weight was measured on an electronic scale, model Profit/UC-321 (Life Source, Milpitas, CA) to the nearest $0.05 \mathrm{~kg}$. Weight was measured at every appointment so that changes could be monitored and controlled in the statistical analysis.

Bone Mineral Density. A Hologic QDR 4500W dual energy x-ray absorptiometry (DEXA) bone densitometer (Waltham, MA) was used to assess BMD at the left hip and spine (anteroposterior view of L1-L4). Measurements $\left(\mathrm{g} / \mathrm{cm}^{2}\right)$ were performed at weeks 0, 8, and 16. Calibrations were performed daily and all measurements and analyses were performed by the same researcher. Analyses were performed using QDR 11.2 scan software (Hologic, Waltham, MA).

Ground Reaction Force. Ground reaction forces were quantified, along with vertical jump height $(\mathrm{VJH})$ and rise rate of force, on a force plate. Subjects jumped onto a $0.51 \mathrm{~m} \times 0.46 \mathrm{~m}$ force plate, model SGA6-4 (AMTI, Watertown, MA) sampling at 1000 Hz. The force plate signal was recorded by a laptop computer, converted from voltage to force, and then normalized to body weight. An average value was calculated from 10 consecutive jumps for peak GRF, VJH, and rise rate of the landing force. Jump height 
Effects of Jumping on Bone Mineral Density in Women 11

was calculated using the equation: height $(\mathrm{m})=1.226 *[\text { flight time }(\mathrm{s})]^{2}$. Rise rate was derived by determining the slope of force versus time from ground contact to peak landing force (4).

Calcium Intake. Dietary calcium and supplemental calcium intake were measured with a Block 98 food frequency questionnaire (Berkeley Nutritional Services, Berkeley, CA). Calcium intake was measured in all groups in order to control for any potential confounding effects of this important mineral.

Menstrual Cycle Questionnaire. A menstrual cycle questionnaire was designed by the investigators and was used to ensure that each subject had a regular menstrual cycle frequency.

Statistical Analysis. All data are expressed as means \pm standard deviations. ANOVA with contrast coding was performed using the general linear model (GLM) technique to determine the extent to which the treatment groups differed from controls in BMD at baseline, 8 , and 16 weeks. ANCOVA was used to reveal mean differences among groups while controlling for age, weight change, calcium intake, ground reaction force, and activity compliance, considered individually and in combination. The Least Squares Means procedure was used to determine specific group mean differences after potential confounding factors were controlled statistically and to provide adjusted means. Alpha was set at the 0.05 level and all calculations were performed using SAS software, version 9.0.

Percent change in BMD was calculated as the baseline BMD level subtracted from the 16 weeks BMD level, divided by baseline BMD level. To decrease redundancy 


\section{Effects of Jumping on Bone Mineral Density in Women}

and to improve readability, "percent change in BMD" is referred to simply as change in BMD throughout the remainder of this paper.

Subjects who did not complete at least $40 \%$ of their prescribed activity were eliminated from the study for noncompliance. For the subjects who completed the study, there was approximately $85 \%$ power to detect an effect size of 0.30 at the 0.05 level.

Because multiple statistical tests were conducted with alpha remaining at the 0.05 level of significance, risk of Type I error across the experiment was inflated. However, if adjustments were made to protect against increased Type I error, then the risk of Type II error would have been increased. The choice was made to prevent an increase in Type II error rather than Type I error.

\section{RESULTS}

The program was completed during 16 weeks from March to July by 64 women, $73 \%$ of the 88 women who started the study. The majority of participants were Caucasian, educated, and married. For the analysis, four women were excluded because of poor compliance, leaving 60 women, 23 subjects in the control group and 23 and 14 subjects in treatment groups Jump 10 and Jump 20, respectively. For all participants, average age was $39.5 \pm 5.46$ years, mean weight was $65.3 \pm 10.3 \mathrm{~kg}$, and average height was $1.65 \pm 0.06 \mathrm{~m}$. Additional group characteristics are listed in Table 1.

\section{Baseline}

There were no significant mean differences among the three groups on any baseline variable (see Table 1). There were no significant differences in initial BMD 
values at any site, age, or weight between subjects who completed the study and those who dropped out.

\section{Weeks}

As shown in Table 2, when age $(\mathrm{F}=4.69, p=0.03)$, weight change $(\mathrm{F}=4.15, p=$ $0.05)$, or compliance scores $(\mathrm{F}=4.63, p=0.04)$ were statistically controlled individually, there were significant differences among groups for hip BMD change. When all covariates were controlled simultaneously, there were differences at the trend level $(\mathrm{F}=$ $3.38, p=0.07$ ) among groups for change in hip BMD. When compared to controls, the Jump 20 group showed greater increases in BMD at the hip $(p=0.05)$.

Also revealed in Table 2, there were no statistically significant differences among groups for change in BMD at the greater trochanter, neck of the hip, or lumbar spine with potential confounders controlled individually or in combination. Baseline calcium intake and GRF were not included in the statistical models displayed in the table, because these variables had no meaningful effects on BMD scores when analyzed.

\section{Weeks}

Compliance to the jumping protocol from week 4 to week 16 for the Jump 10 group was $86.0 \pm 14.8 \%$ and for the Jump 20 group, $85.7 \pm 14.2 \%$, not counting the four subjects assigned to the Jump 20 group who were removed from the study due to poor compliance (compliance $<40 \%$ ).

As shown in Table 3, there were significant differences after 16 weeks for hip BMD change among groups $(\mathrm{F}=7.39, p=0.01)$ with age, weight change, and compliance controlled simultaneously. Specifically, the Jump 20 and Jump 10 groups 
14 Effects of Jumping on Bone Mineral Density in Women

increased $0.51 \%$ and $0.55 \%$, respectively, while controls lost $1.30 \%$ of hip BMD.

Significant differences were revealed between the Jump $20(p=0.02)$ and the Jump 10 $(p=0.01)$ groups compared to controls.

Results were similar for change in greater trochanter BMD at 16 weeks among groups $(\mathrm{F}=5.99, p=0.02)$ with all covariates controlled. The Jump 10 group increased in trochanter BMD by $0.21 \%$, however, the Jump 20 group lost $0.34 \%$. During this period, controls lost $1.76 \%$ of greater trochanter BMD. The Jump 10 group $(p=0.01)$ had significantly greater BMD increases than controls, whereas differences between the Jump 20 group $(p=0.08)$ and controls were less significant.

Also in Table 3, there were significant differences among groups for change in BMD at the neck of the hip with age controlled statistically $(\mathrm{F}=4.31, p=0.04)$. With age, weight change, and subject compliance scores controlled simultaneously, there were no differences among groups regarding changes in BMD at the neck of the hip at 16 weeks. There were no statistical differences for spine BMD change with covariates controlled individually or collectively.

At 16 weeks there were no significant differences between the Jump 10 and Jump 20 groups on any of the performance variables. Specifically, VJH for the Jump 10 group was $37.0 \pm 14.0 \mathrm{~cm}$ and $33.0 \pm 9.0 \mathrm{~cm}$ for the Jump 20 group $(\mathrm{F}=0.57, p=0.46)$. GRF for the Jump 10 group was $380 \pm 95 \%$ body weight (BW) and $401 \pm 169 \%$ BW for the Jump 20 group $(\mathrm{F}=0.24, p=0.63)$, whereas the rise rate of peak force was $217 \pm 343$ $\mathrm{kN} / \mathrm{s}$ for the Jump 10 group and $243 \pm 366 \mathrm{kN} / \mathrm{s}$ for the Jump 20 group $(\mathrm{F}=0.05, p=$ $0.83)$. 
Effects of Jumping on Bone Mineral Density in Women 15

Compliance Scores Restricted to Those $\geq 80 \%$

Further assessment of compliance by eliminating subjects who performed $<80 \%$ of their assigned jumps reduced the number of subjects in each group to 22 and 9 in the Jump 10 and Jump 20 groups, respectively. This additional compliance restriction strengthened the mean change in hip and trochanter BMD significantly. Specifically at eight weeks, hip BMD change was different at the trend level among groups $(F=3.76$, $p=0.06)$ with age, weight change, and compliance statistically controlled. The Jump 10 and Jump 20 groups increased $0.23 \%$ and $1.25 \%$, respectively, while the control group lost $0.59 \%$. Significant differences were present between the Jump 20 group $(p=0.03)$ and controls. There were no other significant differences among groups at the greater trochanter, neck of the hip, or spine for BMD change with all covariates controlled individually or simultaneously.

At 16 weeks, after adjusting for all covariates, there were significant differences among groups for hip $(\mathrm{F}=5.59, p=0.02)$ and trochanter $(\mathrm{F}=9.81, p=0.003) \mathrm{BMD}$ change. The Jump 10 and Jump 20 groups increased hip BMD by $0.50 \%$ and 1.17\%, respectively, while controls decreased hip BMD by $1.06 \%$. These results were significantly different for the Jump 20 group and controls $(p=0.02)$, while the Jump 10 group was significantly different from controls at the trend level $(p=0.10)$.

At 16 weeks, the Jump 10 group increased $0.81 \%$ BMD at the greater trochanter, while the Jump 20 group and controls lost $0.22 \%$ and $2.15 \%$, respectively. Both the Jump $10(p=0.002)$ and Jump $20(p=0.032)$ groups were significantly different than controls. 
16 Effects of Jumping on Bone Mineral Density in Women

\section{Within Group Analysis}

Within group analyses revealed no significant changes in BMD from baseline to 16 weeks for either jumping group. The control group lost significant BMD at the hip $(t=-2.64, p=0.02)$ and greater trochanter $(t=-2.82, p=0.01)$, and the loss was significant at the trend level $(t=-1.92, p=0.07)$ for the neck of the hip. At the spine there were no significant changes from baseline to 16 weeks for controls.

\section{DISCUSSION}

Following 8 weeks of training in premenopausal women, hip BMD changes were significantly greater for subjects in the Jump 20 group compared to controls when age, weight change, or compliance were statistically controlled individually. Differences in hip BMD were greater for the jumpers compared to controls at the trend level, after adjusting for all of the potential confounders simultaneously, suggesting that changes in hip BMD begin to manifest themselves at 8 weeks, especially for those jumping 20 times

per session. These results are similar to the animal studies conducted by Cullen et al. (8) and Umemura et al. (27). Cullen et al. (8) reported increased bone formation in rat tibia after just 6 weeks of mechanical loading. Umemura et al. (27), using a protocol of multiple jump sessions and rest between jumps in rats, stated that bone formation was increased after just 8 weeks. This bone formation was greater in the rats that rested for 30 seconds between jumps than rats that rested for less time. There have been no previous reports of bone changes resulting from jumping at 8 weeks in premenopausal women at the hip or spine. 
The tendency at 8 weeks was for the Jump 20 group to have greater changes in BMD than the Jump 10 group compared to controls. However, BMD for the Jump 10 and Jump 20 groups did not differ statistically. From an effort and time perspective, the Jump 10 group protocol may be better since these subjects spent only half of the time and effort as the Jump 20 subjects, while gaining similarly in BMD.

At 16 weeks, the Jump 10 group tended to have greater changes in BMD than the Jump 20 group compared to controls. Although there were no significant differences in BMD between the treatment groups at 16 weeks, the attrition rate for the Jump 20 group was much greater than for the Jump 10 group. Over the 16-week study, 15 of the Jump 20 subjects dropped out of the study, whereas only six participants from the Jump 10 group quit. These results lend support to the Jump 10 protocol being the most effective protocol.

After 16 weeks of jumping, there were significant differences in hip BMD changes among groups after adjusting for differences in age, weight change, and compliance scores. Specifically, both the Jump 10 and Jump 20 groups had positive hip BMD changes, whereas controls had a negative change in hip BMD. The increases in BMD were similar to those uncovered in studies conducted by Bassey et al. $(3,4)$ and Sugiyama et al. (25).

At the greater trochanter, there were significant differences in BMD change among groups, after statistically adjusting for all covariates at 16 weeks. The Jump 10 protocol resulted in a positive change in greater trochanter BMD, but the Jump 20 group and controls had negative changes. The decrease in greater trochanter BMD is different 


\section{Effects of Jumping on Bone Mineral Density in Women}

than previous studies $(3,4,12,25)$. Because all of the jumps were performed in subjects' homes, the Jump 20 group may have had less motivation to jump maximally each time due to the time and effort requirements. Failure to comply with the protocol could explain the decrease in greater trochanter BMD in the Jump 20 group at 16 weeks.

For the neck of the hip, subjects in the jumping groups had significantly greater BMD changes than controls with age controlled statistically. Specifically, BMD for the Jump 10 group increased significantly more than for controls. However, after adjusting for all of the covariates, there were no significant differences among the groups. Apparently, jumping has little effect on BMD at the neck of the hip in 16 weeks.

Findings for the neck of the hip supports the results of Bassey and Ramsdale (3), but is dissimilar to what has been reported by Heinonen et al. (32) and Bassey et al. (4). The duration of the Bassey et al. (4) and Heinonen et al. (12) studies was 24 weeks, while the Bassey and Ramsdale (3) study was completed after 20 weeks. This may be an indication that the neck of the hip requires additional time than 16 or 20 weeks to manifest significant changes in BMD compared to controls.

Further, in the present study there were no differences in BMD among groups for the lumbar vertebrae. This is similar to findings reported by Bassey et al. $(3,4)$ but was different from the study of Heinonen et al. (12). Heinonen et al. (12) reported a GRF in their treatment group of 5.6 times body weight. The Jump 10 and the Jump 20 groups, along with the studies conducted by Bassey et al. $(3,4)$, reported GRF of 3.8, 4.0, 2.0, and 3.0, respectively. Failure to improve BMD at the spine in the present study and the investigations by Bassey et al. $(3,4)$ may be due to a GRF threshold effect. In short, 
GRF of at least 5.6 times body weight may be needed to significantly increase BMD at the spine.

In general, reported compliance across the 16 weeks was good. Women indicated that they were able to integrate the jumping into their daily routines. Compliance, when used in the statistical models, led to a strengthening of the mean differences at the hip and greater trochanter. Apparently, compliance to the jumping protocols increased significantly the likelihood that BMD would improve, especially at the hip and greater trochanter, whereas poor compliance was predictive of poor BMD outcomes.

Limiting subjects to those with compliance scores showing completion of at least $80 \%$ of their jumps revealed significant differences in BMD at the hip among groups at 16 weeks. Compared to the change in BMD with compliance restricted to at least $40 \%$ of their jumps, greater compliance for the Jump 20 group resulted in greater BMD gains. At eight weeks the results were significant at the trend level. To date, no other study has revealed changes in hip BMD at just eight weeks.

According to the within-group analyses, jumping groups may need more than 16 weeks to experience significant gains in BMD at the hip and greater trochanter. The control group, performing stretching activities, decreased significantly in BMD at the hip and greater trochanter, and showed a significant decrease at the trend level for the neck of the hip. These results suggest that stretching may be detrimental to bone health. Further studies will need to address the effect stretching has on bone density compared to controls. 
20 Effects of Jumping on Bone Mineral Density in Women

A potential limitation of the present study was the lack of an objective measurement of estrogen status. Estrogen has been reported to be protective of bone and it appears that accelerated BMD loss at menopause is due to decreases in estrogen (1). At baseline, subjects completed a questionnaire in order to determine if any subjects were experiencing infrequent menstruation cycles or any symptoms of menopause. All women reported that they had menstruated at least 10 times in the last 12 months and had no symptoms of menopause. Randomization of subjects to groups should have equalized the number of women with low estrogen levels. However, because estrogen levels were not measured, differences among groups could not be checked.

A second limitation was that compliance and adherence to the jumping protocol was self-reported. In addition to jumping logs, compliance was assessed through weekly phone calls. Those that missed jumps were encouraged to complete the full protocol. At the monthly appointment, researchers were able to assess jumping technique in those assigned to the jumping groups. Because compliance was monitored in each subject, differences in compliance could be adjusted statistically in the present study. From the results, the compliance scores proved to be powerful predictors of BMD improvements. Perhaps the biggest limitation of the present study was the large attrition in the Jump 20 group. Each group's sample size was designed to allow $40 \%$ attrition and still maintain adequate power to detect changes in BMD. However, at the conclusion of 16 weeks, $52 \%$ of the Jump 20 group had quit. Reasons for attrition included reoccurrence of previous health problems and lower limb overuse injuries, but lack of interest was the 
Effects of Jumping on Bone Mineral Density in Women 21

largest cause for termination. Apparently, jumping 20 times, twice daily, and waiting 30 seconds between jumps, required too much time and effort from subjects.

Because the study was designed so that all exercise sessions were completed by each subject in her own home, generalization of the results to typical women in society seems appropriate. Subjects reported in this study that they had integrated the jumping protocol into their daily schedules. If subjects would have been required to come to the lab to perform their jumps, greater attrition may have occurred, and generalization of the results would have been more restricted.

A strength of the present study was adjusting for potential confounding factors, such as age, weight change, compliance to the treatment protocol, GRF, and calcium intake. Previous premenopausal, high-impact studies $(3,4,25)$ have not reported any statistical control of potential confounders, while one study (12) controlled only for baseline BMD values. Adjusting for covariates allows for true differences among groups to be revealed, independently of the effect of covariates.

In conclusion, when compared to controls, increases in BMD at the hip and greater trochanter after 16 weeks of high-impact jump training can be achieved by premenopausal women jumping 10 times, twice daily, with 30 seconds of rest between each jump. Likewise, women who consistently perform 20 jumps, twice daily, will tend to increase BMD at the hip compared to controls. 
22 Effects of Jumping on Bone Mineral Density in Women

\section{REFERENCES}

1. Osteoporosis Prevention, Diagnosis, and Therapy. NIH Consensus Statement 2000 March 27-29. 17: 1-45, 2000.

2. Physician's Guide to Prevention and Treatment of Osteoporosis. Belle Mead, NJ: Excerpta Medica, Inc., 1999.

3. Bassey EJ and Ramsdale SJ. Increase in femoral bone density in young women following high-impact exercise. Osteoporos Int 4: 72-75, 1994.

4. Bassey EJ, Rothwell MC, Littlewood JJ, and Pye DW. Pre- and postmenopausal women have different bone mineral density responses to the same high-impact exercise. J Bone Miner Res 13: 1805-1813, 1998.

5. Berard A, Bravo G, and Gauthier P. Meta-analysis of the effectiveness of physical activity for the prevention of bone loss in postmenopausal women. Osteoporos Int 7: 331-337, 1997.

6. Brown JL and Griebler R. Reliability of a short and long version of the block food frequency form for assessing changes in calcium intake. J Am Diet Assoc 93: 784-789, 1993.

7. Creighton DL, Morgan AL, Boardley D, and Brolinson PG. Weight-bearing exercise and markers of bone turnover in female athletes. J Appl Physiol 90: 565$570,2001$.

8. Cullen DM, Smith RT, and Akhter MP. Time course for bone formation with long-term external mechanical loading. J Appl Physiol 88: 1943-1948, 2000. 
9. Dook JE, James C, Henderson NK, and Price RI. Exercise and bone mineral density in mature female athletes. Med Sci Sports Exerc 29: 291-296, 1997.

10. Ernst E. Exercise for female osteoporosis. A systematic review of randomised clinical trials. Sports Med 25: 359-368, 1998.

11. Friedlander AL, Genant HK, Sadowsky S, Byl NN, and Gluer CC. A two-year program of aerobics and weight training enhances bone mineral density of young women. J Bone Miner Res 10: 574-585, 1995.

12. Heinonen A, Kannus P, Sievanen H, Oja P, Pasanen M, Rinne M, Uusi-Rasi K, and Vuori I. Randomised controlled trial of effect of high-impact exercise on selected risk factors for osteoporotic fractures. Lancet 348: 1343-1347, 1996.

13. Heinrich CH, Going SB, Pamenter RW, Perry CD, Boyden TW, and Lohman TG. Bone mineral content of cyclically menstruating female resistance and endurance trained athletes. Med Sci Sports Exerc 22: 558-563, 1990.

14. Kemper HC, Twisk JW, van Mechelen W, Post GB, Roos JC, and Lips P. A fifteen-year longitudinal study in young adults on the relation of physical activity and fitness with the development of the bone mass: The Amsterdam Growth And Health Longitudinal Study. Bone 27: 847-853, 2000.

15. Kroger H, Tuppurainen M, Honkanen R, Alhava E, and Saarikoski S. Bone mineral density and risk factors for osteoporosis--a population-based study of 1600 perimenopausal women. Calcif Tissue Int 55: 1-7, 1994.

16. Mares-Perlman JA, Klein BE, Klein R, Ritter LL, Fisher MR, and Freudenheim JL. A diet history questionnaire ranks nutrient intakes in middle- 
24 Effects of Jumping on Bone Mineral Density in Women aged and older men and women similarly to multiple food records. J Nutr 123: 489$501,1993$.

17. Moreira Kulak CA, Schussheim DH, McMahon DJ, Kurland E, Silverberg SJ, Siris ES, Bilezikian JP, and Shane E. Osteoporosis and low bone mass in premenopausal and perimenopausal women. Endocr Pract 6: 296-304, 2000.

18. Nichols DL, Sanborn CF, Bonnick SL, Gench B, and DiMarco N. Relationship of regional body composition to bone mineral density in college females. Med Sci Sports Exerc 27: 178-182, 1995.

19. Nieves JW, Golden AL, Siris E, Kelsey JL, and Lindsay R. Teenage and current calcium intake are related to bone mineral density of the hip and forearm in women aged 30-39 years. Am J Epidemiol 141: 342-351, 1995.

20. Reid IR, Ames R, Evans MC, Sharpe S, Gamble G, France JT, Lim TM, and Cundy TF. Determinants of total body and regional bone mineral density in normal postmenopausal women--a key role for fat mass. J Clin Endocrinol Metab 75: 45$51,1992$.

21. Robling AG, Burr DB, and Turner CH. Partitioning a daily mechanical stimulus into discrete loading bouts improves the osteogenic response to loading. $J$ Bone Miner Res 15: 1596-1602, 2000.

22. Robling AG, Burr DB, and Turner CH. Recovery periods restore mechanosensitivity to dynamically loaded bone. J Exp Biol 204: 3389-3399, 2001. 
Effects of Jumping on Bone Mineral Density in Women 25

23. Robling AG, Hinant FM, Burr DB, and Turner CH. Improved bone structure and strength after long-term mechanical loading is greatest if loading is separated into short bouts. J Bone Miner Res 17: 1545-1554, 2002.

24. Robling AG, Hinant FM, Burr DB, and Turner CH. Shorter, more frequent mechanical loading sessions enhance bone mass. Med Sci Sports Exerc 34: 196202, 2002.

25. Sugiyama T, Yamaguchi A, and Kawai S. Effects of skeletal loading on bone mass and compensation mechanism in bone: a new insight into the "mechanostat" theory. J Bone Miner Metab 20: 196-200, 2002.

26. Turner CH and Robling AG. Designing exercise regimens to increase bone strength. Exerc Sport Sci Rev 31: 45-50, 2003.

27. Umemura Y, Sogo N, and Honda A. Effects of intervals between jumps or bouts on osteogenic response to loading. J Appl Physiol 93: 1345-1348, 2002.

28. Wolff I, van Croonenborg JJ, Kemper HC, Kostense PJ, and Twisk JW. The effect of exercise training programs on bone mass: a meta-analysis of published controlled trials in pre- and postmenopausal women. Osteoporos Int 9: 1-12, 1999. 
26 Effects of Jumping on Bone Mineral Density in Women

Table 1. Group Characteristics

\begin{tabular}{|c|c|c|c|c|c|}
\hline \multirow[b]{2}{*}{ Variables } & \multicolumn{3}{|c|}{ Groups } & \multirow[b]{2}{*}{$\mathrm{F}$} & \multirow[b]{2}{*}{$p$} \\
\hline & Jump 10 & Jump 20 & Controls & & \\
\hline Age & $41.09 \pm 4.38$ & $39.79 \pm 4.79$ & $37.65 \pm 6.40$ & 2.42 & 0.10 \\
\hline Height, m & $1.65 \pm 0.07$ & $1.67 \pm 0.07$ & $1.64 \pm 0.06$ & 0.79 & 0.46 \\
\hline Calcium, mg & $1059 \pm 657$ & $1004 \pm 491$ & $1109 \pm 538$ & 0.14 & 0.87 \\
\hline Baseline GRF, N & $2448 \pm 71$ & $2571 \pm 67$ & $2206 \pm 74$ & 1.77 & 0.18 \\
\hline Baseline VJH, cm & $34.0 \pm 11.0$ & $35.0 \pm 9.0$ & $31.0 \pm 10.0$ & 0.46 & 0.64 \\
\hline Baseline Weight, kg & $67.88 \pm 9.44$ & $65.10 \pm 11.84$ & $62.88 \pm 10.02$ & 1.37 & 0.26 \\
\hline 8 weeks Weight, kg & $67.54 \pm 9.55$ & $64.69 \pm 11.52$ & $62.30 \pm 9.05$ & 1.63 & 0.21 \\
\hline 16 weeks Weight, kg & $67.96 \pm 9.60$ & $64.60 \pm 11.17$ & $62.02 \pm 8.83$ & 2.16 & 0.13 \\
\hline Baseline Total Hip BMD, mg/cm² & $0.935 \pm 0.081$ & $0.915 \pm 0.079$ & $0.907 \pm 0.088$ & 0.68 & 0.51 \\
\hline Baseline Troch BMD, $\mathrm{mg} / \mathrm{cm}^{2}$ & $0.701 \pm 0.065$ & $0.686 \pm 0.072$ & $0.673 \pm 0.065$ & 1.00 & 0.38 \\
\hline Baseline Neck BMD, $\mathrm{mg} / \mathrm{cm}^{2}$ & $0.814 \pm 0.079$ & $0.809 \pm 0.082$ & $0.832 \pm 0.095$ & 0.43 & 0.65 \\
\hline Baseline Spine BMD, $\mathrm{mg} / \mathrm{cm}^{2}$ & $1.015 \pm 0.083$ & $0.988 \pm 0.098$ & $1.009 \pm 0.064$ & 0.57 & 0.57 \\
\hline
\end{tabular}

Values are means $\pm \mathrm{SD}$; values are unadjusted for potential confounders

GRF: ground reaction force

VJH: vertical jump height

BMD: bone mineral density

Troch: hip greater trochanter

Neck: neck of hip 
Effects of Jumping on Bone Mineral Density in Women 27

Table 2. Comparison of Average Group Percent Change in Bone Mineral Densities Over 8 Weeks

\begin{tabular}{|c|c|c|c|c|c|}
\hline \multirow{2}{*}{$\begin{array}{l}\text { Variable } \\
\quad \text { Variables Controlled }\end{array}$} & \multicolumn{3}{|c|}{ Groups } & \multirow[b]{2}{*}{$\mathrm{F}$} & \multirow[b]{2}{*}{$p$} \\
\hline & Jump 10 & Jump 20 & Controls & & \\
\hline \multicolumn{6}{|l|}{ Total Hip } \\
\hline Unadjusted & $0.40 \pm 1.89^{\mathrm{a} \S}$ & $0.83 \pm 2.17^{\mathrm{a}}$ & $-0.58 \pm 1.75^{\mathrm{b}}$ & 5.41 & 0.02 \\
\hline Age & $0.38^{\mathrm{a}, \mathrm{b}}$ & $0.83^{\mathrm{a}}$ & $-0.55^{b}$ & 4.69 & 0.03 \\
\hline WtChng & $0.28^{\mathrm{a}, \mathrm{b}}$ & $0.86^{\mathrm{a}}$ & $-0.47^{b}$ & 4.15 & 0.05 \\
\hline Comp & $0.43^{\mathrm{a} \S}$ & $0.88^{\mathrm{a}}$ & $-0.64^{b}$ & 4.63 & 0.04 \\
\hline Age, WtChng, comp & $0.29^{\mathrm{a}, \mathrm{b}}$ & $0.91^{\mathrm{a}}$ & $-0.51^{b}$ & 3.38 & 0.07 \\
\hline \multicolumn{6}{|l|}{ Trochanter } \\
\hline Unadjusted & $-0.28 \pm 2.20$ & $0.07 \pm 2.04$ & $-0.77 \pm 2.50$ & 1.26 & 0.27 \\
\hline Age & -0.36 & 0.05 & -0.68 & 0.74 & 0.39 \\
\hline WtChng & -0.30 & 0.07 & -0.76 & 1.15 & 0.29 \\
\hline Comp & -0.17 & 0.20 & -0.96 & 2.61 & 0.16 \\
\hline Age, WtChng, comp & -0.26 & 0.22 & -0.89 & 1.54 & 0.22 \\
\hline \multicolumn{6}{|l|}{ Neck of Hip } \\
\hline Unadjusted & $-0.29 \pm 3.06$ & $0.35 \pm 3.56$ & $-0.82 \pm 2.50$ & 1.12 & 0.25 \\
\hline Age & -0.13 & 0.38 & -1.00 & 1.86 & 0.18 \\
\hline WtChng & -0.37 & 0.36 & -0.75 & 0.83 & 0.37 \\
\hline Comp & -0.21 & 0.45 & -0.96 & 1.33 & 0.25 \\
\hline Age, WtChng, comp & -0.19 & 0.43 & -0.97 & 1.32 & 0.26 \\
\hline \multicolumn{6}{|l|}{ Spine } \\
\hline Unadjusted & $-0.01 \pm 2.70$ & $-0.51 \pm 2.34$ & $-0.43 \pm 1.87$ & 0.07 & 0.79 \\
\hline Age & -0.02 & -0.51 & -0.43 & 0.06 & 0.81 \\
\hline WtChng & -0.09 & -0.50 & -0.36 & 0.01 & 0.92 \\
\hline Comp & 0.14 & -0.33 & -0.70 & 0.69 & 0.41 \\
\hline Age, WtChng, comp & 0.03 & -0.31 & -0.60 & 0.39 & 0.74 \\
\hline
\end{tabular}

Note: Means in the same row with the same superscript letter are not significantly different, $p>0.05$

Values are average percentage point change \pm SD. On rows showing variables controlled, change scores are adjusted for differences in the covariate(s).

WtChng: weight difference, weight at 16 weeks - weight at baseline

Comp: mean compliance of week 4 through week 16

${ }^{\S}$ The Jump 10 group and Controls were different at the trend level, $0.05<p<0.10$ 
28 Effects of Jumping on Bone Mineral Density in Women

Table 3. Comparison of Average Group Percent Change in Bone Mineral Densities Over 16 Weeks

\begin{tabular}{|c|c|c|c|c|c|}
\hline \multirow[b]{2}{*}{$\begin{array}{l}\text { Variable } \\
\quad \text { Variables Controlled }\end{array}$} & \multicolumn{3}{|c|}{ Groups } & \multirow[b]{2}{*}{$\mathrm{F}$} & \multirow[b]{2}{*}{$p$} \\
\hline & Jump 10 & Jump 20 & Controls & & \\
\hline \multicolumn{6}{|l|}{ Total Hip } \\
\hline Unadjusted & $0.28 \pm 1.62^{\mathrm{a} \S}$ & $0.23 \pm 3.02^{\mathrm{a}, \mathrm{b}}$ & $-0.85 \pm 1.55^{\mathrm{b}}$ & 4.22 & 0.04 \\
\hline Age & $0.24^{\mathrm{a} \S}$ & $0.22^{\mathrm{a}, \mathrm{b}}$ & $-0.82^{\mathrm{b}}$ & 3.50 & 0.07 \\
\hline WtChng & $0.33^{\mathrm{a}}$ & $0.22^{\mathrm{a}, \mathrm{b}}$ & $-0.90^{\mathrm{b}}$ & 4.60 & 0.04 \\
\hline Comp & $0.58^{\mathrm{a}}$ & $0.53^{\mathrm{a}}$ & $-1.32^{\mathrm{b}}$ & 7.96 & 0.01 \\
\hline Age, WtChng, comp & $0.55^{\mathrm{a}}$ & $0.51^{\mathrm{a}}$ & $-1.30^{\mathrm{b}}$ & 7.39 & 0.01 \\
\hline \multicolumn{6}{|l|}{ Trochanter } \\
\hline Unadjusted & $-0.12 \pm 2.50$ & $-0.59 \pm 1.23$ & $-1.27 \pm 2.16$ & 2.50 & 0.12 \\
\hline Age & -0.19 & -0.61 & -1.19 & 1.78 & 0.19 \\
\hline WtChng & $0.02^{\mathrm{a}}$ & $-0.62^{\mathrm{a}, \mathrm{b}}$ & $-1.40^{\mathrm{b}}$ & 3.79 & 0.06 \\
\hline Comp & $0.16^{\mathrm{a}}$ & $-0.29^{a^{*}}$ & $-1.74^{\mathrm{b}}$ & 5.72 & 0.02 \\
\hline Age, WtChng, comp & $0.21^{\mathrm{a}}$ & $-0.34^{\mathrm{a}^{*}}$ & $-1.76^{\mathrm{b}}$ & 5.99 & 0.02 \\
\hline \multicolumn{6}{|l|}{ Neck of Hip } \\
\hline Unadjusted & $0.44 \pm 3.85$ & $0.35 \pm 1.93$ & $-0.93 \pm 2.32$ & 2.81 & 0.10 \\
\hline Age & $0.64^{\mathrm{a}}$ & $0.39^{\mathrm{a}, \mathrm{b}}$ & $-1.15^{\mathrm{b}}$ & 4.31 & 0.04 \\
\hline WtChng & 0.47 & 0.34 & -0.96 & 2.88 & 0.10 \\
\hline Comp & 0.39 & 0.30 & -0.85 & 1.44 & 0.24 \\
\hline Age, WtChng, comp & 0.59 & 0.26 & -1.03 & 2.83 & 0.15 \\
\hline \multicolumn{6}{|l|}{ Spine } \\
\hline Unadjusted & $0.01 \pm 1.74$ & $-0.58 \pm 2.44$ & $-0.52 \pm 1.92$ & 0.18 & 0.67 \\
\hline Age & -0.08 & -0.60 & -0.42 & 0.02 & 0.89 \\
\hline WtChng & -0.11 & -0.56 & -0.41 & 0.02 & 0.89 \\
\hline Comp & -0.11 & -0.71 & -0.33 & 0.01 & 0.90 \\
\hline Age, WtChng, comp & -0.28 & -0.65 & -0.19 & 0.17 & 0.69 \\
\hline
\end{tabular}

Note: Means in the same row with the same superscript letter are not significantly different, $p>0.05$

Values are average percentage point change \pm SD. On rows showing variables controlled, change scores are adjusted for differences in the covariate(s).

WtChng: weight difference, weight at 16 weeks - weight at baseline

Comp: mean compliance of week 4 through week 16

${ }^{*}$ The Jump 20 group and Controls were different at the trend level, $0.05<p<0.10$

${ }^{\S}$ The Jump 10 group and Controls were different at the trend level, $0.05<p<0.10$ 
Figure 1. Mean Percent Change in Hip BMD Over 16 Weeks

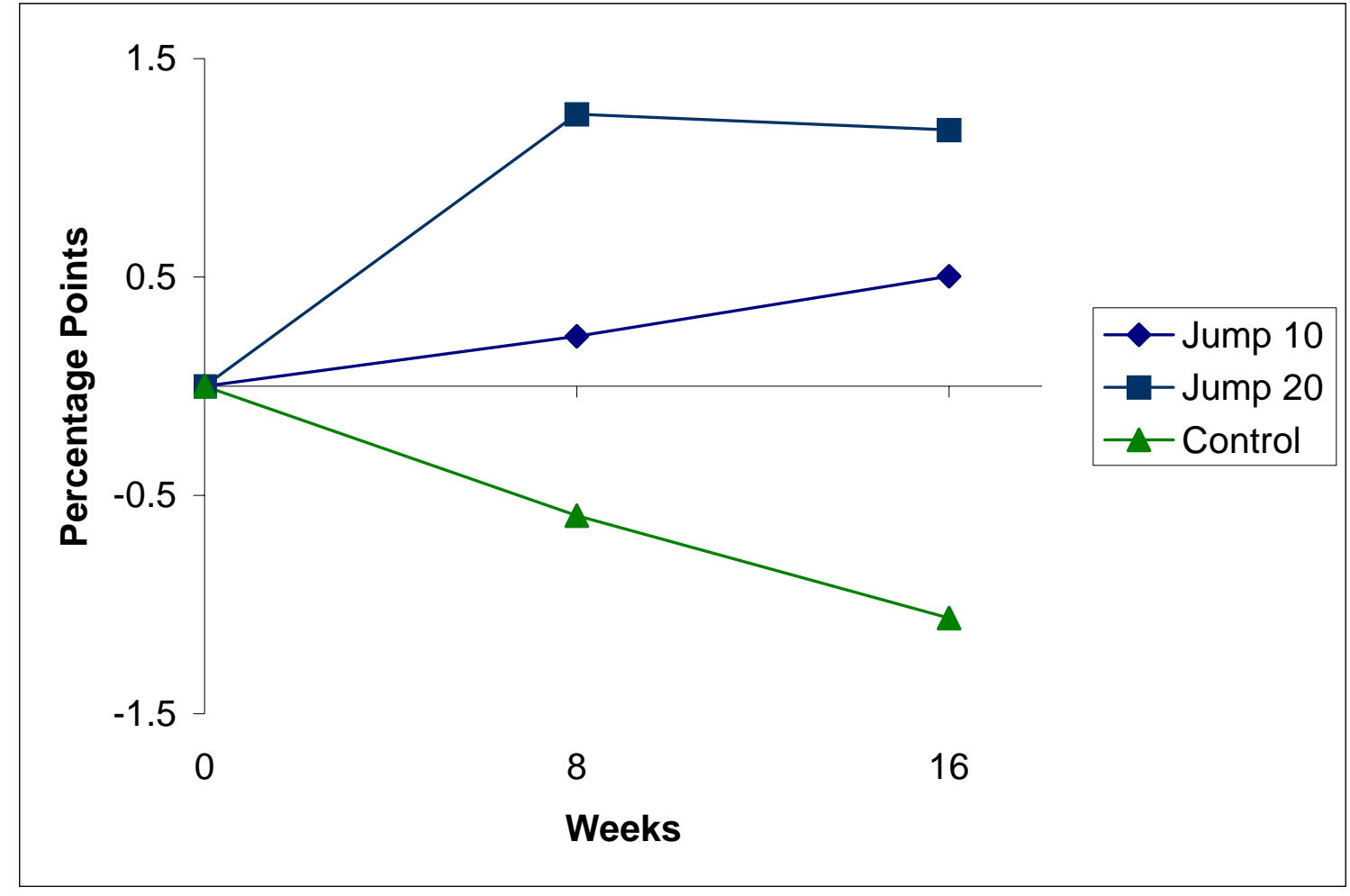

Includes only those subjects that performed at least $80 \%$ of the assigned protocols

All covariates were controlled

At 16 weeks, hip BMD changes for the Jump 20 group were significantly greater than changes in controls $(p=0.02)$

At 16 weeks, the Jump 10 group was significantly greater at the trend level compared to controls $(p=0.10)$

There were no significant differences in BMD between the jump groups 
30 Effects of Jumping on Bone Mineral Density in Women

Figure 2. Mean Percent Change in Trochanter BMD Over 16 Weeks

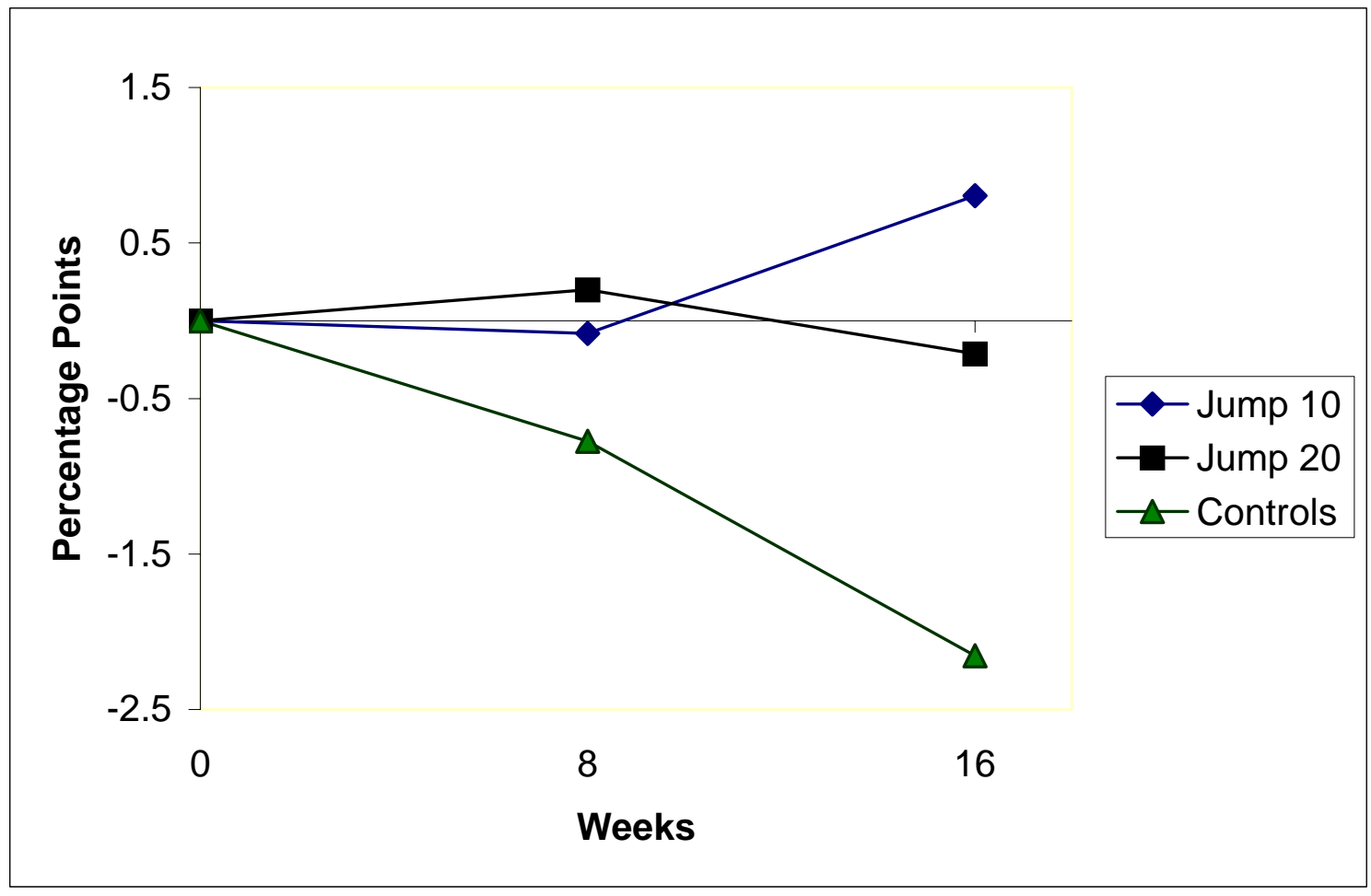

Includes only those subjects that performed at least $80 \%$ of the assigned protocols

All covariates were controlled

At 16 weeks, changes in trochanter BMD for the Jump $20(p=0.032)$ and Jump 10 $(p=0.002)$ groups were significantly greater than for controls

There were no significant differences in BMD between the jump groups 
Appendix A

Prospectus 


\section{Chapter 1}

Introduction

Bone is living connective tissue, which gives support and protection to the body. During adolescence, bone mass is greatly increased with $98 \%$ of the maximum being added by age $20(2,38)$. Specific regions, like the femoral neck and lumbar vertebrae, may finish adding bone mass at an even earlier age $(12,38,55)$. After bone reaches peak mass in women, it slowly declines until perimenopause $(38,55,78)$.

Peak bone mass or density is a major factor in determining the strength of bone. Research has found that bone mineral density accounts for $70 \%$ of total bone strength (1). Bone quality is another factor in determining the ultimate strength of bone. Quality includes the bone's geometry, or cross-sectional area (61). Increased density around the cross-sectional area of bone will increase the strength of bone. This strength is important in relation to the risk of fracture (78). A decrease in either the density or geometry of the bone will increase the risk of fracture. Density is lost as a result of age causing an increased risk of fracture (28). When bone loss is accelerated or if there is a sub-optimal peak bone mineral density (BMD), osteoporosis can develop (47).

Osteoporosis is a disease characterized by an increased fracture risk due to decreased bone content. It results in increased morbidity and mortality along with financial, psychosocial, and physical costs (2). The most common fracture sites associated with osteoporosis are the hip and lumbar vertebrae. Each year there are approximately 700,000 and 300,000 lumbar vertebrae (spine) and hip fractures, respectively, within the United States (2). Osteoporotic fractures have been estimated to 
cost $\$ 10-15$ billion annually in direct health care expenses, with hip fractures incurring the greatest costs $(1,2)$. Psychosocially, the cost of impact on the individual and family is immeasurable. Many hip fracture patients develop secondary complications such as depression, pressure ulcers, pneumonia, or a reduction in individual freedom following the fracture (67).

The probability that a white woman over age 50 will have a hip fracture is $14 \%$ (1). It was reported in the third National Health and Nutrition Education Survey that there were 10 million women suffering from osteoporosis at the hip and 19 million people with low BMD at the hip in the United States (2). One estimate states that by the year 2040, the number of hip fractures could triple in the United States (2). This poses a serious threat to the health care system unless osteoporosis can be prevented.

Prevention of osteoporosis is mostly a function of addressing the risk factors that a woman possesses. Age, race, and genetics are examples of nonmodifiable risk factors. Modifiable risk factors include estrogen deficiency and lifestyle-related behaviors such as smoking, excessive alcohol intake, poor calcium consumption, and a sedentary lifestyle. Since most women in the U.S. do not abuse tobacco or alcohol (14), estrogen deficiency, calcium intake, and physical inactivity have been the risk factors where prevention efforts have focused.

Measurement of a woman's estrogen status requires blood tests, and if the levels are low, may require hormone replacement therapy. This may be impractical or undesirable for many women due to costs or health care issues (17). However, calcium intake and physical activity do not require medical attention in order to make changes. 
Nutrition intake provides the building blocks that the body needs to ensure optimal mineralization of bone. Calcium is the most plentiful mineral in bone. Without proper calcium intake, bones are subjected to parathyroid hormone, which break down bone in order to increase calcium levels (101).

Physical activity, on the other hand, provides a stimulus to bone to increase mineralization in areas of new stress (42). This new stress is a direct result of the activity chosen (42). To be strengthened, bone must be subjected to strains to which it is not accustomed. Activities of daily living in non-sedentary individuals are not capable of increasing BMD, because the strains do not reach the osteogenic strain threshold (99).

Exercise, a subset of physical activity, may be capable of generating strains that cross the osteogenic strain threshold. Exercise can be divided into impact and nonimpact activities based on the loading pattern. Both types of exercise have muscle contractions producing strains on bone, but ground reaction forces (GRF) are only involved with impact exercise. Non-impact exercise, such as biking or swimming, have not been effective in changing BMD at the hip $(19,22,34)$ or spine $(19,34)$. Impact activities, like running, weight lifting, and certain sporting events such as basketball, volleyball, or gymnastics, have produced increases in hip BMD due to the large GRF (10, $24,51,93,99)$. Due to impact activities, positive BMD changes have been seen in adolescents, premenopausal, and postmenopausal women. (4, 5, 22, 30, 39, 78, 93, 99, 100).

Jumping is also an impact exercise and several research projects have studied its effect on hip and spine BMD. These studies $(7-9,15,27,32,96)$ have focused 
specifically on the magnitude of GRF, the number of repetitions performed, and the number of times a session is performed.

In most jumping studies, GRF have been reported to be between 2.0 and 9.0 times body weight $(7-9,15,27,32,96)$. Jumping repetitions have varied among studies with some subjects jumping rope for a certain amount of time or a specific number of jumps. Other studies have required between 33 and 100 jumps in one jumping session $(7-9,15$, $27,29,32,33,77,79,96-98)$. The number of sessions that subjects performed each week varied from 3-7 times per week $(7-9,15,27,29,32,33,77,79,96-98)$. Results of these studies have been mixed for both hip and spine BMD for adolescents, premenopausal, and postmenopausal women $(7-9,15,27,29,32,33,77,79,96-98)$.

Research on BMD and jumping, in rats, has also focused on the frequency of jumps and the magnitude of GRF. Findings from rat studies have raised the question of whether or not a delay between jumps and/or sets might promote increased BMD.

Bone has been found to lose sensitivity to the loading stimulus after the first couple of jumps $(36,45,88)$. If a stimulus from a jump occurs before the bone restores sensitivity, the bone may have a diminished response or no response at all $(69,71)$. Research has determined that shorter exercise sessions of high magnitude strain followed by rest between repetitions and sets may be more beneficial than one long exercise session each day (68-71).

\section{Statement of the Problem}

The purpose of this proposed research project is to determine the effects of four different jumping protocols involving differences in jump sessions and repetitions on hip 
and spine BMD. Secondary objectives will be to determine if changes in hip and spine BMD are independent of age, body weight, initial BMD, calcium intake, and ground reaction force within the sample.

\section{Research Questions}

1. To what extent is hip BMD increased as a result of differences in jump sessions and repetitions at 8 and 16 weeks?

2. To what extent do the jumping groups differ in hip BMD at 8 and 16 weeks?

3. To what extent is spine BMD increased as a result of differences in jump sessions and repetitions at 8 and 16 weeks?

4. To what extent do the jumping groups differ in spine BMD at 8 and 16 weeks?

5. To what extent do confounding factors, like age, body weight, initial BMD, calcium intake, and ground reaction force, influence the relationship between jumping treatment and BMD at the hip and spine?

Definitions

Bone Mineral Density (BMD) - The amount of mineralized tissue in the bone. It is used as a surrogate measure for bone strength, which can predict fracture risk.

Bone Quality - Refers to the architecture, turnover, damage accumulation, and mineralization of bone (1).

Dual-Energy X-ray Absorptiometry (DEXA or DXA) - a diagnostic test to measure BMD using two $\mathrm{x}$-ray energies.

Exercise - Planned, purposeful activity designed to improve fitness. 
Ground Reaction Force (GRF) - The force that the ground exerts on the individual as they land from a jump. Can be standardized and reported in multiples of body weight.

Osteogenic Response - Anabolic response of bone to stimuli above a certain threshold with a certain amount of rest time needed before full sensitivity is again achieved in the bone.

Physical Activity (PA) - Any activity that increases the metabolism of the body above resting values.

Knee Bends - Simulated squatting motion using just body weight as the resistance. Correct form involves keeping the knees from crossing the big toe by pushing out the buttocks.

Bone Remodeling - The interactions of osteoclasts resorbing bone and osteoblasts laying down new bone.

Osteogenic Index - Method to quantify and compare exercise regimens based on intensity, number of repetitions, number of sessions per day, and the recovery time between multiple sessions.

Vertical Jump Height (VJH) - The maximum vertical height obtained from jumping on a flat surface.

\section{Assumptions}

Validity of this study will rely on the following assumptions:

1. Subjects will perform the jumping protocol correctly. 
2. Subjects will perform the assigned number of jumps and jump sessions, six days per week.

3. Subjects will report their nutritional and supplement intake on the food frequency questionnaire accurately.

4. Subjects will not change their eating behavior during the study.

5. Subjects will not perform any vigorous exercise during the study.

6. Subjects' left hip accurately portrays the right hip BMD.

7. Menstrual cycle frequency will not change during the study.

\section{Limitations}

Limitations of the study include subjects not complying with the exercise or control group protocols, changing their exercise or nutritional habits, under or overreporting their calcium intake, incorrectly answering questionnaires, or completely dropping out of the study.

\section{Delimitations}

Data collection will commence in 2004 and will include 120 women between the ages of 30-45 years. Subjects will be primarily white, married, non-smokers, and nonobese. Left hip and lumbar vertebrae BMD will be measured by DEXA (Hologic, Bedford, MA). Calcium intake and supplement use will be assessed through the Block 98 food frequency questionnaire (Berkeley Nutritional Services, Berkeley, CA). Menstrual cycle regularity will be assessed through a written questionnaire. Lastly, GRF will be assessed using a force plate (AMTI, Watertown, MA). 


\section{Chapter 2}

\section{Review of Literature}

Recent findings have suggested that impact exercise may be one of many factors essential to increase bone mineral density (BMD). This review will be limited to examining the literature that investigates the hip and spine BMD of women across the lifespan. Topics will include the effects of general physical activity, strength training, running, strength training combined with running, sport activity that involves high-impact loading, and jumping, as covered in meta-analyses, cross-sectional, experimental, and longitudinal research. Finally, bone mechanosensitivity will be discussed, followed with a conclusion of the literature review.

General Physical Activity

Physical activity (PA) is any movement that increases metabolism. For this part of the review, general PA will include all activity that is not considered high impact exercise, specifically weight lifting, aerobic training, a combination of weight lifting and aerobic training, or high impact sports.

Adolescence offers the female body the best opportunity to increase bone mass. This stems from an increase in sex hormones following menarche (43). The effects of PA can be confounded by the increase in sex hormones, changes in nutrition, and changes in weight (49). Most studies involving children or adolescents have examined the effects of exercise or impact loading on BMD, instead of generalized PA.

Lack of activity and its association with peak bone mass was investigated by Wang et al. (94). Their retrospective study used black and white female children 9-18 
years of age. Results showed an inverse relationship between a sedentary lifestyle and peak bone mass after controlling for race, weight, and height.

Several longitudinal studies have been conducted to study the effects of generalized PA on BMD in adolescents. A prospective study of prepubertal children was conducted over a three-year period. At the conclusion, it was discovered that girls in the highest quartile of PA exhibited 20\% greater femoral neck BMD than the lowest PA quartile (43). Results were similar in another prospective study using adolescent children. The study was conducted for six years and again found that girls in the top quartile of PA had significantly greater bone mass for the femoral neck and spine than girls in the lowest quartile of PA (43).

Several cross-sectional studies $(3,47,76,81,92,102,103)$ have been conducted investigating the relationship between levels of PA and hip and spine BMD. Results from these studies have all shown a positive relationship-the greater the participation in PA the greater the BMD. A drawback of these studies, however, has been the method used to measure PA. All but one study has used questionnaires to assess PA. Zhang et al. used personal activity monitors in order to have an objective measurement of PA. A unique result of this measurement was that increases of $100 \mathrm{kcal} /$ day in energy expenditure resulted in an increase of $12 \mathrm{mg} / \mathrm{cm}^{2}$ bone density at the spine (102).

There have been mixed results in premenopausal women for PA and BMD using longitudinal studies. A study conducted for 15 years investigated the relationship between PA and hip and spine BMD. The study started when the subjects were adolescents and finished when they reached 28 years of age. It was found that activity 
during adolescence was positively correlated with hip and spine BMD. These results were independent of age, body composition, and calcium intake (42).

Conflicting results were found with two longitudinal studies. Both studied premenopausal women between 20-40 years and found no relationship between levels of PA or fitness and hip or spine $\operatorname{BMD}(53,90)$.

The effectiveness of PA on hip and spine BMD of postmenopausal women was assessed through a recent meta-analysis (10). Only prospective studies with a control group were included. In addition, inclusion criteria required that PA was the only intervention examined. After applying the criteria, 18 studies from 1978 to 1995 were reviewed. Results of the analysis revealed that general PA did not have an effect on femoral BMD. However, PA seemed to prevent spinal BMD loss in postmenopausal women (10).

Like premenopausal women, cross-sectional research of PA using postmenopausal women has been positive for hip and spine $\operatorname{BMD}(6,18,56,62,65,80$, 95). Moreover, three studies $(56,62,95)$ have assessed muscle strength and found a positive relationship between strength and hip BMD. Another study of postmenopausal women showed that cardiorespiratory fitness is positively correlated with hip BMD (65).

The association between muscle strength and BMD was corroborated with three longitudinal studies in postmenopausal women. In one study, physically active women were able to maintain muscle strength and thus had greater hip BMD than inactive women (91). It was also revealed that physically inactive women tended to lose more BMD than active women (57). Feskanich et al. concluded that women with no other 
activity than walking four hours per week were $41 \%$ less likely to have a hip fracture than those who only walked one hour per week (25).

In summary, the effects of general PA on hip and spine BMD have been mixed. Cross-sectional studies have found positive relationships between general PA and hip and spine BMD. Some longitudinal studies have not found a positive association between PA and hip and spine BMD. A common problem with PA studies has been the method of assessing PA, which has been self-reported for all but one study. This has likely weakened the relationship between general PA and hip and spine BMD. Strength Training

Strength training is considered an exercise that may impose a load great enough to go above bone's osteogenic threshold. Kelley et al. performed a meta-analysis (41) to examine the effect strength training has on the bone of women. Strict inclusion criteria were used. The studies included in the meta-analysis employed a control group or subjects acted as their own controls before starting the training protocol. Only studies with subjects who were at least 18 years old were included. Studies were also required to include hip and spine BMD measurements and last at least 16 weeks. After applying the above inclusion criteria, 29 studies qualified between the years 1966 and 1998 .

The primary outcomes for the above studies were changes in BMD at the femur and spine. Quality scores were given to each study after reviewing its randomization, blinding, and report of withdrawal or dropouts. Effect sizes were also computed for each BMD region, along with a check for any publication bias. 
There was no evidence for publication bias for either the spine or femur. Changes in BMD at the femur were statistically insignificant. However, subgroup analysis revealed significant differences in femoral BMD for postmenopausal women. The spine showed a small, but significant, change in BMD between treatment and control groups with no menopausal status association.

Strength training in young women has elicited a positive relationship with BMD at the spine and femur compared to inactive young women $(21,34)$. The subjects utilized were body builders who spent between 6-10 hours each week weight lifting. These results cannot be associated with regular young women due to the time and intensity of the body building workouts. Moreover, this type of research can only show relationships between weight lifting and BMD and not causality. Intervention studies are needed to provide evidence of a causal relationship.

A weight lifting intervention study (59) using adolescent females looked at changes in BMD at the hip and spine. The intervention lasted 15 months with each subject lifting three days per week for an average of 30 minutes each session. Sets, repetitions, and weight were progressively increased across the study. At the conclusion of the study, adolescent females showed significant increases in BMD only at the femoral neck.

The authors reported that the dropout rate for the treatment group was approximately $85 \%$. Those remaining in the treatment group had a compliance of $73 \%$. It is possible that significance was not seen in other areas due to the lack of statistical power. 
A recent intervention study (50) involved older women with an average age in the low 50s. Subjects assigned to the treatment group strength trained for 24 weeks. At the conclusion of the study, no differences in BMD were found at the hip or spine. The study did show trends for an increase in both hip and spine BMD in the training group.

Maddalozzo and Snow stated that lack of statistical power and utilizing women less than three years past menopause were major limitations of the study. This study also used the subjects as their own controls with the control period lasting only 12 weeks. This was only half the length of the treatment intervention.

Humphries et al. (37) conducted a similar study for 24 weeks. Subjects were assigned to a treatment group that strength trained, while a control group walked twice per week. At the conclusion of the intervention, there was no significant difference between groups for hip and spine BMD.

Limitations to the study conducted by Humphries were $61 \%$ statistical power to detect significance as well as having controls walk twice a week. The walking could have decreased the treatment effect of weight training between groups. Another potential confounding variable may have been menopause status. Perimenopausal women were unequally divided between the treatment and control groups.

In summary, cross-sectional research has shown positive relationships between strength training and hip BMD compared to inactive females. Experimental research studying the effect of strength training on hip and spine BMD has been mixed for women. Lastly, meta-analysis research (41) has indicated that strength training tends to 
result in small, favorable effects for postmenopausal women at the hip and for all women at the spine.

Running

Running provides a stimulus to bone in the form of muscle contractions as well as ground reaction forces (GRF). The magnitude of the GRF is determined by the speed at which a subject runs, and it has been found that the GRF for running in women is generally between two to three times their body weight (BW) (19).

Cross-sectional studies have examined the relationship between running and BMD in both younger and older women. Studies with younger women have also compared college runners versus recreational runners with results being mixed for BMD at the hip and spine.

Heinrich et al. (34) studied recreational and collegiate runners and found no significant difference in BMD at the hip or spine between them and non-runners. However, Wolman et al. (100) found that runners versus non-runners had increased bone content within the femoral shaft, whereas Creighton et al. (19) showed that after controlling for body mass index, body weight, and nutrition, runners had greater BMD at the greater trochanter of the hip compared to non-runners. Differences may be due to the lack of control of potential confounders in the Heinrich et al. study (34) or the different bone measurement area in the Wolman et al. study (100).

Kirk et al. (44) looked at the relationship of $\mathrm{VO}_{2 \max }$ and spine $\mathrm{BMD}$ in runners compared to non-runners. They found that within premenopausal runners there was a positive correlation between spine $\mathrm{BMD}$ and $\mathrm{VO}_{2 \max }$, while within postmenopausal 
runners there was not a significant correlation. In contrast, Shimegi et al. (72) found that postmenopausal Japanese joggers had greater BMD at the spine compared to inactive women.

Causality of changes in BMD by running was addressed by Dalsky et al. (20). These researchers set up an intervention in postmenopausal women that lasted for 22 months and included a control group. The treatment group exercised for 50-60 minutes, three times per week at $70-90 \%$ of their $\mathrm{VO}_{2 \max }$. At the end of nine months, there was a $5.2 \%$ increase in spine BMD, and after 22 months the increase jumped to $6.2 \%$.

Some subjects were asked to reduce or stop their training following the ninemonth measurement. At the end of the 22 months, it was seen that these subjects had returned to near baseline values in spinal BMD. The authors concluded that reducing or stopping treatment will reverse any benefits that the treatment had on BMD.

A significant limitation of this study was that subjects were not randomly assigned to groups. This makes it difficult to know if the treatment effects were due to the training or due to selection bias.

Two longitudinal studies $(31,54)$ investigated the effects of long-term running on hip and spine BMD. Both projects used postmenopausal women and lasted for approximately five years. Both studies found that women who maintained their running programs maintained BMD at the hip and spine. This was manifested in Z-score improvement compared to non-runners during the study. For runners that decreased their training, greater bone loss occurred compared to those who did not decrease their training, due to the reduction in stimulus to the bone (54). Significant positive 
correlations were found for the percentage change in BMD and average minutes run per week, $r=0.66$, along with BMD change and percent change in minutes run per week, $r=0.54(54)$.

In summary, the relationships between running and hip and spine BMD have been mixed in cross-sectional studies using premenopausal women. However, longitudinal studies in postmenopausal women have reported positive relationships between runners who have maintained their training programs and spinal BMD. Causality of increased spinal BMD from running has been supported by one intervention study in postmenopausal women. However, more research is needed before the relationship between running and hip and spine BMD can be considered causal.

Strength Training and Aerobic Exercise

A cross-sectional study (21) examined the relationship between strength training supplemented with aerobic exercise and spinal BMD. A sedentary group, doing less than one hour of exercise per week, was compared to a group that performed aerobic exercises and strength training. Subjects were young women with an average age of 24 years. The analysis showed that participation in strength training and aerobic exercise was related to increased spinal BMD when compared to non-exercisers. The type of aerobic exercise completed by subjects was not included in the study, which may have influenced the results.

In order to evaluate causality between strength training coupled with aerobic exercise and BMD, three intervention studies $(26,46,63)$ have been completed. Young women, with an average age of 29 years, were used by Friedlander et al. (26) to 
investigate changes in BMD at the hip and spine resulting from strength training combined with aerobics. This study randomly assigned subjects to a control or strength training (ST) and endurance-training group (ET). The intervention lasted two years. Strength, $\mathrm{VO}_{2 \max }$, and $\mathrm{BMD}$ were measured at baseline, midtest, and posttest.

The control group performed stretching exercises two times per week for 30 minutes. This design was implemented in order to decrease attrition from the control group. The treatment group's protocol changed with each exercise session during the week. The first session was comprised of circuit training, which incorporated highimpact aerobics. The second session was comprised of only ST, while the third session used only high-impact aerobics.

At the one-year midtest, only strength and $\mathrm{VO}_{2 \max }$ were significantly different between the exercise and the control groups. At the conclusion of the study, changes in strength, $\mathrm{VO}_{2 \max }$, and $\mathrm{BMD}$ at the femoral neck, greater trochanter, and spine were significantly greater for the exercisers than in the controls.

Neither the intensity of weight training nor how subjects progressed in their workouts was reported. The reported range of weights available for subject use was 3-12 pounds and 16-36 pounds for dumbbells and barbells, respectively. The aerobic training included high impact exercises performed once a week. The combination of activities makes it difficult to ascertain if the changes in BMD at the hip and spine were due to ST, or to the impact of the aerobic sessions, or to the combination of both activities.

Peterson et al. (63) studied older women enrolled in either an ET, ST+ET, or a control group. The ST+ET group completed their resistance training at home using free- 
weights, training three times per week. The endurance portion of the study was completed at the research center and was comprised of 50 minutes of endurance dancing, performed three times per week. Intensity for the endurance dancing was set at $75-80 \%$ of subjects' heart rate reserve. After 12 months, posttest measurements were performed on strength, and bone mineral density of the hip and spine.

Only shoulder flexion and extension muscle torque in the ST+ET were different than controls. Bone density was not different between the control, ET, and ST+ET groups at the hip or spine. The authors concluded that the lack of change could have been from too light of a load placed upon the spine and hip from weight training. There were trends for increased strength and BMD, but they were not significant at 12 months.

The authors did not include a power analysis to show their ability to detect significant changes, nor was menstrual status of subjects reported. This could have been a confounding factor, since the average age of subjects was 53 years and some subjects may have been pre-, peri-, or postmenopausal. Another critical detriment of this study was that subjects were able to self-select the group they wanted to participate in.

Kohrt et al. (46) examined women 10 years older on average than those used by Peterson et al. (63). The intervention study lasted 11 months and random assignment of subjects to groups was completed by matching subjects by weight. The treatment group performed strength training and rowing exercises, whereas the control group only completed normal activities of daily living. Following training, the spine and Ward's triangle region of the hip were significantly greater than controls based on percentage increase of BMD. 
These results of the Kohrt et al. investigation differed from the Peterson et al. study. This may be partially explained by the increased age of subjects. Kohrt et al. reported that subjects had experienced menopause at least 10 years earlier. This would allow for BMD to stabilize after the accelerated loss associated with menopause before treatment was utilized.

In summary, a positive relationship between a combination of strength training and running on hip and spine BMD was reported in one study. Intervention studies, however, have been mixed in their results. This may be partially explained by the different ages and protocols used by the researchers.

High Impact Sport Activity

Weight lifting, running, or a combination of both, generally produce GRF less than four times BW $(19,23)$, which has led to the investigation of several high-impact sports and their effect on BMD. Fuchs et al. quantified medium impact activities as those with GRF between two and four times BW, while high impact activities were those with GRF above four (27). Volleyball, basketball, rope skipping, and gymnastics have been studied as high impact activities. Their impact, or GRF, have been quantified to be between 4.1-6.0 times BW for basketball and volleyball and as high as 8.0 for gymnastics $(19,27)$.

The high-impact of these sports has been positively associated with greater BMD at the spine and hip when compared to controls $(19,39,58,64,72)$. Cross-sectional studies specifically examining women athletes have found significance at the spine (19, $58,64)$, greater trochanter $(19,64)$, and femoral neck $(19,58)$, even after controlling for 
weight $(19,58)$, BMI, and nutrition (19). Two studies have investigated the effect of volleyball on hip and spine BMD in pre-, peri-, and postmenopausal women. Both studies found that women who continued to play the sport had greater hip and spine BMD than controls $(39,72)$.

In summary, all research on high-impact sporting activities has shown a positive relationship between participation in the activities and hip and spine BMD. The drawback to this research is that it has all been cross-sectional in design, and causality cannot be concluded. It is also not clear as to what aspects of the activities are responsible for the positive relationship with BMD.

Jumping

Jumping has recently been researched as an exercise intervention to increase hip and spine BMD. Studies have been conducted in prepubescent children, adolescent children, premenopausal women, and postmenopausal women. Each study has used a different jumping protocol, and so the present literature review will focus on each study individually.

Prepubescent children were randomized to a treatment or control group for a seven-month intervention trial (27). The treatment group jumped off $61 \mathrm{~cm}$ high boxes, which produced a GRF of about 8.8 times BW on landing. A total of 100 jump-offs were performed during three sessions each week, while controls performed non-impact stretching. Exercise sessions were led by a researcher and performed as part of a normal school routine. During the middle of the study, subjects missed three weeks due to school vacation. 
Repeated measures ANCOVA controlled for age, baseline bone values, and height and weight change scores. The analysis revealed that the treatment group had significantly greater increases in the femoral neck and spine bone mineral content (BMC) (27) compared to the control group.

A quasi-experimental study performed with adolescents used plyometric jumping as the intervention for nine months (98). High school freshman girls were recruited to participate in a PE class in order to do the jump training. Controls were then recruited and matched to the treatment group by age and months past menarche. Training took place three times per week on various surfaces, and used various jumps. The training was progressive with the girls starting with 140 jumps per session and proceeded to over 360 jumps at the end of training. Attendance to the PE classes was reported at $86 \%$ for the nine-months.

Results showed the only significant difference between the treatment group and the controls was at the greater trochanter of the hip. The authors stated that controls participated in twice the physical activity outside of school as the treatment group did. Also, the study was not randomized, which was justified by the researchers in order to increase compliance (98).

Bassey et al. (7) conducted a study with premenopausal women for six months. Subjects were randomized into treatment and control groups. Subjects attended weekly exercise classes that lasted one hour. Controls did all of the low impact exercises and were allowed to walk or swim at home. The control group was considered a placebo exercise group, whereas the treatment group performed approximately 33 jumps of high- 
impact at the weekly class, along with 50 jumps per day at home. The ground reaction force for the jumps at home was 2.0 times BW.

Bone density at the greater trochanter was greater in the jumping group compared to controls after six months, while the femoral neck approached significance with $\mathrm{p}=$ 0.06. Total subjects were 27 for both groups and the authors did not report the statistical power available to detect changes.

Bassey et al. (9) performed another jumping intervention with a different protocol. Subjects in the treatment group completed 50 jumps per day, six days per week. Jumps were performed at a frequency of $1 \mathrm{~Hz}$, which produced a GRF of 3.0 times BW for the average jump height of $8.5 \mathrm{~cm}$. The study lasted five months and reported $76 \%$ compliance to the exercise protocol.

The treatment group increased their BMD at the femoral neck and greater trochanter compared to controls. However, the greater trochanter at the hip was the only location to achieve statistical significance. No differences were seen between the treatment group and controls for spinal BMD. Power was reported to be $95 \%$ to find differences of $2.0 \%$ in BMD.

Heinonen et al. (32) conducted an 18-month intervention study with premenopausal women who were randomly assigned to a jumping group or a control group. Jumpers performed progressive high-impact exercises three times per week. As the exercises were progressed, GRF increased from 2.1 to 5.6 times BW. All training sessions were supervised and as training progressed, the number of jumps decreased from 
200 initially to only 100 per session at the end of the study. Training compliance was reported as $83 \%$.

After adjusting for baseline values, the jump group had significant increases in BMD over the controls at the femoral neck and spine. Differences in spine BMD results in this study as compared to that of Bassey et al. $(7,9)$ could have been due to the differences in peak GRF. At the end, the jump groups in the Heinonen et al. study were producing almost twice the GRF as those produced in the Bassey et al. studies.

A continuation of the Heinonen et al. study (33) revealed that a reduced training protocol over eight months could still elicit increased BMD values at the femoral neck compared to controls. Values at the spine decreased from where they were at the completion of the 18-month study. In the follow-up study, jumps were completed only two times per week and produced only 2.0-4.5 times BW in GRF.

Apparently, the femoral neck is able to continue to increase in BMD even after a reduction in GRF. However, the reduced GRF was not high enough to sustain spinal BMD changes. This lends further support regarding why Bassey et al. $(7,9)$ did not find any BMD changes at the spine. The largest GRF of the continuation study (33) was 50$125 \%$ greater than the GRF produced in the Bassey et al. studies $(7,9)$. This reduction in GRF resulted in a decrease in spinal BMD for subjects in the treatment group.

A six-month study was conducted with pre and postmenopausal Japanese women (79). Subjects were able to self-select participation in the treatment or control group. The treatment group was instructed to jump rope 100 times per day with three days separating each jumping session. Controls were asked to only participate in activities 
they were currently doing. Neither GRF nor overall compliance of the treatment protocol was reported.

Premenopausal women who were in the treatment group increased total hip and femoral neck BMD compared to the controls, whereas spinal BMD was not changed significantly. Postmenopausal women had no significant changes in BMD at any site. Femoral neck and total hip BMD showed a trend towards significance, but only 13 subjects were used in the treatment group and no power statistics were provided. Results should be interpreted with caution, due to the self-selection of subjects into the two groups.

As part of the publication reporting on premenopausal women (9), Bassey et al. conducted a randomized, controlled trial of postmenopausal women over the course of 12 months, followed by 6 months of follow-up. Women in the treatment group performed 50 jumps per day, 6 days per week (9). At the completion of the study, postmenopausal women did not have any significant changes at either 12 or 18-months, despite a GRF of 4.0 times BW and 69\% compliance.

Welsh et al. (97) conducted a study with nine postmenopausal women in a treatment group. Subjects in the treatment group met at a YMCA and participated in 40 minutes of aerobic work, which included 6-7 minutes of jumping or skipping. Controls used in the study were age and menopausal status matched to those in the treatment group. The intervention lasted for 12 months with compliance reported at $80 \%$. Significant differences were reported between the treatment group and control group. The treatment group increased their femoral neck BMD, while controls had a 
decrease in BMD at the same site. There were trends for increases in BMD at the Ward's triangle and the greater trochanter, but they were not significant. The authors neglected to report the statistical power available to see differences between groups.

A similar study (29) using postmenopausal women examined the difference in low-impact and high-impact activities compared to controls regarding spine BMD for one year. Subjects were matched by weight and then randomly assigned to the three groups. Treatment groups participated in a supervised exercise program, three times per week for one hour. The low-impact group performed exercises that produced GRF less than 2.0 times BW, such as slow walking, heel jacks without a jump, and the charleston. Highimpact exercises were classified as those producing GRF in excess of 4.0, like running in place, jumping jacks, and knee-to-elbow jumping. Average GRF was reported to be 1.49 and 3.49 times BW for the low and high-impact treatment groups, respectively.

After one year, subjects in the treatment groups had maintained their spinal BMD compared to controls who lost BMD. There were no differences between the two exercise groups for BMD. The lack of difference between the treatment groups could be due to the fact that the average peak GRF for high-impact exercise was only 3.49 times BW. Previous studies $(7,9)$ that have investigated spinal BMD have not seen increases when impact forces have been that low.

The above results are similar to those found in a study conducted by Bassey and Ramsdale (8). Their subjects in a randomized treatment group did 50 heel drops per day. This produced a GRF of 2.5 times BW. After 6 and 12 months, no differences were found between controls and the treatment group in spinal or hip BMD. A statistical 
power analysis was omitted from the methods and only 20 subjects were used in each of the groups, treatment and control.

Two studies $(77,96)$ have combined the effects of high-impact exercise with weight lifting to determine their effects on hip and spine BMD. Specifically, a study involving young women with an average age of 24 years assessed the effect of impact exercise on BMD (96). Subjects were randomized into an exercise or control group. The exercise group completed three sessions of weight lifting along with 60 minutes per week of rope jumping for 24 months. At the end of the study, hip BMD decreased more in the exercise group than the controls, while spinal BMD was not significantly different between groups (96).

Snow et al. (77) conducted a study similar to Weaver et al. (96), except that they used postmenopausal women and a weighted vest while jumping. Subjects performed the exercise intervention for five years and at the conclusion, those in the exercise group had greater BMD in all subregions of the hip compared to controls. Some limitations that the authors listed were that some subjects were using hormone replacement therapy and that subjects were not initially randomized to research groups.

In summary, results of jumping studies have been mixed for hip and spine BMD. It appears that pre- and postmenopausal women react differently to the stimulus placed on bone from jumping. Randomized, experimental studies in premenopausal women have found significance at the hip, whereas spine BMD may be dependent on a GRF of at least five times BW. Studies conducted on postmenopausal women have resulted in 
mixed results at the hip and spine without regard to reported GRF. The discrepancies in postmenopausal results may be due to a lack of protocol standardization.

Bone Mechanosensitivity

Many researchers have studied the effects of exercise on bone. They have worked on hypotheses of how bone changes in relation to exercise stimulus. Three hypotheses have been proposed in order to optimize osteogenesis. First, the load on bone must be dynamic in nature. Second, loading is required for only a short duration, and third, bone will accommodate to routine loads and thereby decrease the responsiveness of bone to those loads (84).

Bone, at the microscopic level, consists of osteocytes surrounded in fluid and connected to other osteocytes via canaliculi (13). It has been proposed that the fluid movement within bone is what is responsible for the osteogenic response $(13,83,85)$. In order for fluid movement to occur, bone must be subjected to cyclic loading and relaxation $(13)$

Several studies have been conducted using animals, which have provided valuable insights regarding bone mechanosensitivity. In review of some earlier animal studies, Turner found that dynamic loads stimulated osteogenesis. By increasing the magnitude of dynamic loading, the signal for osteogenesis was further optimized. Static loads, however, even with increased magnitude, do not tend to stimulate osteogenesis. These findings have given support to the fluid theory of bone growth (84).

Another important part of dynamic loading is how quickly loading achieves peak GRF, or strain rate. This strain rate may be more influential on bone growth than the 
magnitude of strain (13). Turner et al. (86) researched the impact of strain rate on rats using a four-point bending device. The rats were divided into four treatment groups and received treatment for two weeks. Peak loading was kept the same between each group while load rate was the independent variable.

After the two weeks, the rats in the highest rate of loading had significantly greater bone formation than those with lesser rates. The authors commented that the amount of bone formation was directly proportional to the rate of strain placed on the bone (86).

Judex et al. (40) worked with roosters to study the effects of magnitude and strain rate on BMD using walking and drop-jumping. Maximal compressive strains from dropjumps were $30 \%$ greater than those during walking, while there was a $740 \%$ difference in strain rate between the two activities. Jumps were performed for 3 weeks and bone formation was increased in the jumping group over the control group at the end of the training period.

Along with magnitude of strain and strain rate, another important principle associated with osteogenesis is the duration of bone loading. The number of repetitions is critical to the sensitivity of bone. Umemura et al. (88) studied the effects of the number of jumps performed each day on bone mass in rats. Rats were assigned to a control group or groups that jumped 5, 10, 20, 40, or 100 times per day, five days per week. Jumps consisted of rats jumping up to and pulling themselves onto a ledge $25 \mathrm{~cm}$ high. Jumps were progressed to a $40 \mathrm{~cm}$ ledge. Training lasted for eight weeks. 
At the conclusion of the study, it was discovered that all treatment groups had increased their bone mass. The groups jumping 10, 20, 40, and 100 times were significantly greater than controls, whereas the five jump group showed only a positive trend. There was not any difference in bone mass among those animals that jumped 10, 20,40 or 100 times (88).

Similar results were found for roosters (84), which allowed Turner et al. to derive a mathematical equation for the desensitizing of bone with larger loading repetitions. Bone tissue sensitivity was found to be proportional to $1 /(\mathrm{N}+1)$, where $\mathrm{N}$ is equal to the number of repetitions performed. After the 20th repetition, bone was $95 \%$ insensitive to further loading (87). Mechanosensitivity may be restored to bone with specific periods of rest between loading sessions $(68,71)$.

Robling et al. (71) examined how bone formation changed by separating the number of loading cycles into many different loading sessions. Rats were assigned to two loading groups and two control groups. The loading groups received 360 loading repetitions in one session or 90 repetitions in four sessions separated by three hours rest. Both treatments resulted in increased bone mass, but bone mass in the group performing multiple sessions was significantly greater than the single session group (71).

A follow-up study was conducted by Robling et al. (68), investigated whether or not bone formation was improved by separating loading cycles into one, two, four, or six bouts. Total repetitions were kept constant among groups, while the recovery period decreased from 6 hours in the 2-bout group to 2 hours in the 6-bout treatment group. 
All treatment groups exhibited greater bone formation than the control group. The four and six session groups were significantly greater than the one-bout group, whereas the four and six session groups were not significantly different from each other. The authors concluded that two to three hours appeared sufficient to restore bone's sensitivity to loading (68).

Robling et al. (69) assessed the impact of varying recovery times between multiple loading sessions. Rats were assigned to six loading groups or a control group. Four loading sessions were used for each treatment group. There was either no recovery time between loading sessions or $0.5,1,2,4$, or 8 hours between sessions. Total loading repetitions were kept constant among treatment groups.

Results showed that both the 4 and 8-hour recovery groups had significantly greater bone formation than the no recovery group. The 8-hour recovery group also had greater bone formation than the 0.5 hour recovery group. There were no significant differences between the 1, 2, 4, and 8-hour recovery groups. The authors plotted the results of bone formation as a result of recovery time and found a negative accelerating logarithmic curve. After extrapolating the curve past eight hours, the authors hypothesized that eight hours were required to restore full mechanosensitivity to bone (69).

An eight-week study by Umemura et al. (89) contradicted the benefits of recovery time between loading sessions. For the study, rats were separated into three treatment groups and a control group. Treatment groups performed either single bouts of 20 repetitions or two bouts of ten repetitions (D3). Three seconds of rest separated each 
loading repetition for D3 and one single session group (S3). The other single bout group separated repetitions by 30 seconds (S30). Jumps were performed five days per week with rats jumping up to a $25 \mathrm{~cm}$ ledge, which progressed to $40 \mathrm{~cm}$ after four weeks.

Following training, S3 and D3 had significantly greater changes in bone weight compared to controls. However, there was no difference in bone weight change between S3 and D3 groups.

The authors speculated that the bone for the D3 group accommodated to the loading activity prior to the completion of the study. This partially explains why the D3 group had increased bone weight over the controls, but not over S3 (89). The type of loading was another difference between the studies. Robling et al. $(68,69)$ used a bending device while Umemura had the rats jump up to a ledge. This form of jumping only produced a muscle contraction stimulus to increase bone while the bending device applied loads as great as what is seen with high-impact activity.

A secondary objective of the Robling et al. study (69) was to examine the effect that differing rest periods between repetitions had on bone. Groups received 0.5, 3.5, 7 , 14 seconds rest between repetitions. The group that rested 14 seconds between repetitions had significantly greater bone formation than the other groups. There were no significant differences in bone formation among groups resting $0.5,3.5$, and 7 seconds between repetitions. The authors concluded that it is important to separate repetitions with at least 14 seconds rest in order to aid in the restoration of bone's mechanosensitivity (69). 
Similar results were also found in the Umemura et al. study (89). The treatment group that separated repetitions by 30 seconds had greater bone changes than the S3 and D3 groups.

A review of mechanical properties required for bone formation addressed bone accommodation (84). It was concluded that bone would adapt to the strains placed upon it. If progressive overload is not implemented, bone will only maintain levels achieved and will decrease if the stimulus is withdrawn. Due to this accommodation, activities of daily living have not been shown to be effective in increasing bone in active individuals (48). According to Umemura et al., dynamic loads must be performed in an unusual loading pattern in order for bone to perceive the full stimulus (89).

Turner and Robling addressed the amount of time required between multiple loading sessions to restore bones sensitivity (87). They derived an equation to show the percent restoration of bone mechanosensitivity; sensitivity $(\%)=100\left(1-\mathrm{e}^{-\mathrm{t} / \tau}\right)$, where $\tau$ is approximately six hours. This was then applied to what the authors termed, the osteogenic index (OI).

This index is a way to quantify the stimulus placed on bone. For weekly calculations, it requires the GRF of the activity, the number of repetitions performed, the number of sessions used each day, the recovery time between sessions, and the number of days per week the activity will be performed. It was found that multiple loading sessions per day with at least six hours between sessions of the same GRF would produce a greater OI, and hence a greater stimulus on bone, than just one session per day. 
Animal research has provided insight on how exercise stimulus to bone may be optimized. Research to date has focused on the number of loading repetitions, the number of loading sessions, the optimal rest time between loading sessions, as well as the optimal rest time between repetitions. Research has found that fewer loading repetitions may be more beneficial than many repetitions, whereas multiple loading sessions may be more beneficial than just one. Rest between loading sessions and repetitions are important considerations, since bone requires time between repetitions and sessions in order to be able to fully respond to exercise stimuli.

Conclusion

General physical activity, weight lifting, aerobic exercise, and a combination of weight lifting and aerobic exercise, have produced inconsistent effects on hip and spine BMD. Studies assessing the effects of jumping on hip and spine BMD have had some design problems, including no randomization, insufficient statistical power, or both. Jumping studies that have randomized subjects to a control or treatment group have also had mixed results for spine and hip BMD.

Within premenopausal women, jump results have been positive for hip BMD compared to controls. For spinal BMD, results have been mixed, probably due to design problems. Studies have not been consistent in GRF, number of training days per week, or the numbers of jump repetitions employed. Studies have not reported the rest period used or have had subjects perform jump repetitions in succession. In addition, studies have not tried multiple jumping sessions per day. These variations have delayed the development 
of an optimal exercise protocol that will produce significant increases in BMD at the hip and spine.

Animal studies have shed some light on how an optimal exercise program should be developed to promote gains in BMD. Positive results have been found using fewer loading repetitions, splitting total loading cycles into multiple jumping sessions with at least six to eight hours between sessions, and allowing for short rest intervals between each repetition.

The purpose of this proposed research project will be to determine the effects of four different jumping protocols on hip and spine BMD. Differences within the protocols will include the number of loading sessions and the number of jump repetitions. The proposed study will also include relatively long rest (i.e., 30 seconds) periods between jump repetitions in order to incorporate the animal study findings on rest between loading. 


\section{Chapter 3}

Methods

\section{Design}

The study will use a pretest-midtest-posttest randomized-groups design with four treatment groups and one control group. The independent variable will be the jumping protocol assigned to each treatment group. The dependent variable will be bone mineral density (BMD) at the hip and lumbar vertebrae (spine). The BMD midtest and posttest will occur at 8 and 16 weeks, respectively. Potential confounders will include age, body weight, initial BMD values, calcium intake, and ground reaction force (GRF).

Subjects

A total of 120 premenopausal women, 30-45 years old, will be recruited for the study through newspaper advertisements, flyers, referrals, and phone calls. Each potential subject will be screened for possible exclusion from the study with a short survey (see Appendix B-3). Subjects will be excluded if they have been diagnosed in the last 12 months with a surgical or medical condition that would affect BMD (thyroid or parathyroid disorder, renal or hepatic dysfunction, digestive disorder) (66) or affect exercise compliance (arthritis, back pain, heart condition, or malignancy). Exclusion will also include vigorous exercise (i.e., jogging, strength training) within the last 12 months (women who walk for more than 30 minutes per day and more than five times per week will be excluded), individuals who have lost or gained greater than 10 pounds within the last six months, current dieters, smoking or alcohol consumption, body mass index (BMI) $\geq 25 \mathrm{~kg} / \mathrm{m}^{2}$, age younger than 30 or greater than 45 years, or a hip BMD t-score greater 
than 1.0. Irregular (varying cycle length) or infrequent menstrual cycles (less than 10 cycles per year) (19), a hysterectomy without hormone replacement, or a hysterectomy with hormone replacement within the last six months will lead to exclusion of the volunteer. Users of glucocorticoids, anti-convulsant, or thiazide diuretic medication (66) within the last six months will be excluded, along with women who are pregnant, breastfeeding within the previous six months, or having delivered a baby within the previous 12 months. Qualified subjects will be randomly assigned to one of four treatment groups or a control group.

Research Group Protocols

Subjects in the treatment groups will participate in one of four jumping programs, whereas control subjects will participate in a stretching program. All participants will perform a warm-up of three minutes of brisk walking followed by ten knee bends. All study-related exercise will be completed in the subject's home.

For subjects in the treatment groups, jumps will be performed without shoes and socks. Subjects will jump vertically as high as possible from a hard surface covered with a thin cushion provided by the researchers. The cushion will standardize the thickness that subjects could jump on as well as increase compliance by decreasing the mild discomfort of landing on a hard floor. After landing, subjects will rest for 30 seconds between jumps. Jumping sessions will be performed six days per week according to the protocol of the jump group.

A progression of jump repetitions will be included in order to condition the subject's bodies to the impact of treatment. Initially, subjects in the treatment groups will 
jump from the floor five times for each session that is to be performed during the first week. During the second week, jumps from the floor will increase to 10 for each session for all treatment groups. By the second week, two treatment groups will be performing their total number of repetitions (10 repetitions). Beginning with the third week, the other two treatment groups will perform 15 jumps for each session, which will be increased to 20 jumps in each session by the fourth week. By the beginning of the fourth week, all groups will be performing the maximal number of jumps as set forth in the following protocols:

Jump group 1. Subjects will stand on a mat covered hard surface and jump as high as possible. After landing, the subjects will rest for 30 seconds before performing the next jump. Subjects will complete 10 jumps at each daily session. The full protocol will begin at the start of the second week.

Jump group 2. Subjects will stand on a mat covered hard surface and jump as high as possible. After landing, the subjects will rest for 30 seconds before performing the next jump. Subjects will complete 20 jumps at each daily session. The full protocol will begin at the start of the fourth week.

Jump group 3. Subjects will stand on a mat covered hard surface and jump as high as possible. After landing, the subjects will rest for 30 seconds before performing the next jump. Subjects will complete 10 jumps at each session with at least eight hours separating the two daily sessions. The full protocol will begin at the start of the second week. 
Jump group 4. Subjects will stand on a mat covered hard surface and jump as high as possible. After landing, the subjects will rest for 30 seconds before performing the next jump. Subjects will complete 20 jumps at each session with at least eight hours separating the two daily sessions. The full protocol will begin at the start of the fourth week.

Control group. Stretching has not been shown to successfully promote osteogenic activity. Engaging in a regular stretching program will foster the perception of full participation in the study, which should decrease attrition from the study. Subjects within the control group will perform three thigh stretches for a total of ten minutes per day, six days per week. Subjects will be instructed to stretch a specific muscle group until mild discomfort occurs within that muscle. Subjects will then hold this stretch for 30 seconds before switching to stretch the opposite leg.

The first stretch will work on the hamstring muscle group. The subject will sit on the ground with one leg straight and the other bent at the knee to allow the sole of the foot to touch the inside of the opposite thigh. By leaning forward, the stretch will increase the flexibility of the straight leg without affecting BMD of the hip or spine.

The second stretch will involve the quadriceps. Each subject will lie on the floor on one side and pull the ankle of the top leg back toward the buttocks. Care should be taken that the pull is from above the ankle joint and not pulling on the foot.

The third stretch will involve the hip adductors. Standing with the feet wide apart, subjects will bend one knee and lean to that side. The opposite leg will remain 
straight. Leaning to one side will stretch the inside of the leg that remains straight. Subjects will hold the stretch for 30 seconds before moving to the next stretch.

Procedures

Advertising and subject recruitment for this proposed project will start in 2004. Subjects will be screened for exclusion after they respond to the newspaper advertisements, flyers, referrals, and phone calls. Those who are not excluded will be scheduled for an orientation meeting, which will correspond to either treatment or control group assignment. The study will be approved by the Internal Review Board at BYU. The orientation meeting will provide an overview of what each subject will be required to do over the 16-week study. Subjects will sign an informed consent document and then fill out a menstrual cycle questionnaire (see Appendix A-3). Upon completion of the questionnaire, subjects will sign up for their pretest appointment.

Subjects will receive a food frequency questionnaire at the group assignment meeting along with instructions regarding how to complete the questionnaire. Subjects will be instructed to bring the questionnaire back when they come for their first data collection appointment. A researcher will check the food frequency questionnaire for any areas left blank and will call the subject to obtain any information that is missing.

Data will be collected at baseline, 4, 8, 12, and 16 weeks. At baseline, 8, and 16 weeks, subjects will be weighed, have a hip and spine BMD scan, and perform 10 jumps on a force plate with 30 seconds separating each jump. The appointment will take approximately 30 minutes total. At weeks 4 and 12, subjects will perform 10 jumps on a 
force plate with 30 seconds rest between jumps and be weighed. These appointments will take approximately 15 minutes total.

Data collection will take place in the Human Performance Research Center (HPRC) at Brigham Young University. Subjects will change into shorts and a tee shirt before each data collection session. At the conclusion of each data collection session, subjects will sign up for the next month's appointment. An exercise log will also be given to subjects so they can record the number of jumps and sessions performed each day for the month between data collections (see Appendix A-4). Completed logs will be returned at the subject's next appointment. Subjects will also call weekly and leave a message about their jumping activity. If a subject does not report in, a phone call will be made to check on the subject's activity.

Instrumentation and Measurement Methods

Anthropometric Measures. Measurement of stature and body weight will take place at the orientation meeting and will be used to calculate BMI. Stature will be measured with a stadiometer, model 439 (Cardinal Scale Manufacturing, Webb City, MO). Measurement will take place with subjects standing with their weight distributed evenly and standing with an erect posture. Height will be measured to the nearest $0.1 \mathrm{~cm}$.

Weight will be measured at each data collection appointment. Instruction will be given to not eat or drink anything for three hours prior to their appointment. Subjects will be weighed in shorts and a tee-shirt on an electronic scale, model Profit/UC-321 (Life Source, Milpitas, CA) following the use of the restroom. Body weight will be measured and recorded to the nearest $0.05 \mathrm{~kg}$. Body weight will be collected in order to 
assess any body weight changes due to the treatment protocols or subject behavior. Body weight change may confound the BMD results and therefore will be controlled statistically.

Bone Density. A Hologic QDR 4500 dual energy x-ray absorptiometry (DEXA) bone densitometer (Waltham, MA) will be used to assess BMD at the left hip and spine. DEXA has been shown to be a valid and reliable method for measuring bone mass and density $(16,35,74,75)$. Measurements $\left(\mathrm{g} / \mathrm{cm}^{2}\right)$ will be performed at weeks 0,8 , and 16 by a licensed radiology technician. The DEXA machine will be calibrated before each day's scans and the same investigator will perform the scans and analyses (73). Analyses will be performed using QDR 11.2 scan software (Hologic, Waltham, MA).

Prior to testing, subjects will be asked (1) if they are pregnant, (2) if they have had any active isotopes injected in the last two weeks, and (3) if they have had any surgeries in the last two weeks. Any questions that are answered "yes," will eliminate the subject from the study. Upon answering "no" to the above three questions, the subject will then be positioned on the DEXA scan bed for a spine BMD scan.

To ensure accuracy, a square foam box will be placed under the legs of subjects in order to flatten the spine. Positioning of the DEXA scan arm will be assisted with a laser emitted from the arm. For the spine scan, the crosshairs of the laser will bisect the body vertically and lay one inch below the highest level of the iliac crest.

Positioning for the hip scan will put the crosshairs bisecting the left thigh at the level of the crotch. Positioning will also include medial rotation of the thigh along with 
slight abduction of the leg to minimize the appearance of the lesser trochanter and allow the femoral shaft to be straight on the scan image, respectively.

Ground Reaction Force. Quantification and change of impact forces will be achieved by measuring GRF. Jump height, GRF, and rise rate of force will be measured in the HPRC biomechanics lab at weeks 0,8 , and 16 . Subjects will jump onto a $0.51 \mathrm{~m}$ x $0.46 \mathrm{~m}$ force plate, model SGA6-4 (AMTI, Watertown, MA) sampling at $1000 \mathrm{~Hz}$. The force plate signal will be recorded by a laptop computer and converted from voltage to force and then normalized to body weight. An average value will be calculated for each subject from 10 consecutive jumps for peak GRF, jump height, and rise rate of the landing force. Jump height will be calculated, using the equation: height $(\mathrm{m})=1.226 *[\text { flight time }(\mathrm{s})]^{2}$. Rise rate will be taken from the slope of the force/time relation over $10 \mathrm{~ms}$ on the steepest part of the slope between landing and peak force (9).

Subjects will perform the jumps using the same jumping protocol as performed at home. Subjects will be taught to bend slightly at the hips, knees, and ankles and use their arms for countermovement, allowing for maximal jump height. Subjects will be instructed to land on the balls of their feet, followed by a heel strike. Hips, knees, and ankles will be bent to a midway position between sitting and standing, in order to slightly dampen the impact force. A researcher will assess compliance to the jumping instruction and corrections will be given if a deviation is detected.

Calcium and Supplement Intake Questionnaire. Calcium and supplement intake will be measured with a Block 98 food frequency questionnaire (Berkeley Nutritional Services, Berkeley, CA), which has been validated and has produced reliable results when 
compared to other assessment methods $(11,52,60)$. The questionnaire will be administered one time at the start of the study. Calcium intake has the potential to confound BMD results (82). Measurement of calcium intake will allow for any differences among groups to be controlled statistically.

Menstrual Cycle Questionnaire. A menstrual cycle questionnaire will be designed by the investigators and will be used to ensure that each subject has a regular menstrual cycle frequency.

Statistical Analysis. To determine the extent to which group means differ in BMD over the three data collection periods, repeated measures ANOVA will be employed. ANCOVA using the multiple regression technique will be used to reveal differences among groups while controlling for potential confounders, considered individually and in combination. The Least Squares Means procedure will be used to determine specific group mean differences, before and after the potential confounding factors are controlled statistically. Alpha will be set at the 0.05 level and all calculations will be performed using SAS software, version 9.0.

The 120 women recruited for this study will provide $93 \%$ power to detect an effect size of 0.30 at the 0.05 level. Allowing for $25 \%$ attrition from the treatment groups, power will be $81 \%$ to detect an effect size of 0.30 at the 0.05 level. 


\section{References}

1. Osteoporosis Prevention, Diagnosis, and Therapy. NIH Consensus Statement 2000 March 27-29. 17: 1-45, 2000.

2. Physician's Guide to Prevention and Treatment of Osteoporosis. Washington, DC: National Osteoporosis Foundation, 1999.

\section{Alekel L, Clasey JL, Fehling PC, Weigel RM, Boileau RA, Erdman JW, and}

Stillman R. Contributions of exercise, body composition, and age to bone mineral density in premenopausal women. Med Sci Sports Exerc 27: 1477-1485, 1995.

4. Alfredson H, Nordstrom P, and Lorentzon R. Bone mass in female volleyball players: a comparison of total and regional bone mass in female volleyball players and nonactive females. Calcif Tissue Int 60: 338-342, 1997.

5. Andreoli A, Monteleone M, Van Loan M, Promenzio L, Tarantino U, and De Lorenzo A. Effects of different sports on bone density and muscle mass in highly trained athletes. Med Sci Sports Exerc 33: 507-511, 2001.

6. Astrom J, Ahnqvist S, Beertema J, and Jonsson B. Physical activity in women sustaining fracture of the neck of the femur. J Bone Joint Surg Br 69: 381-383, 1987.

7. Bassey EJ and Ramsdale SJ. Increase in femoral bone density in young women following high-impact exercise. Osteoporos Int 4: 72-75, 1994.

8. Bassey EJ and Ramsdale SJ. Weight-bearing exercise and ground reaction forces: a 12-month randomized controlled trial of effects on bone mineral density in healthy postmenopausal women. Bone 16: 469-476, 1995. 
9. Bassey EJ, Rothwell MC, Littlewood JJ, and Pye DW. Pre- and postmenopausal women have different bone mineral density responses to the same high-impact exercise. J Bone Miner Res 13: 1805-1813, 1998.

10. Berard A, Bravo G, and Gauthier P. Meta-analysis of the effectiveness of physical activity for the prevention of bone loss in postmenopausal women. Osteoporos Int 7: 331-337, 1997.

11. Brown JL and Griebler R. Reliability of a short and long version of the block food frequency form for assessing changes in calcium intake. J Am Diet Assoc 93: 784-789, 1993.

12. Buchanan JR, Myers C, Lloyd T, Leuenberger P, and Demers LM. Determinants of peak trabecular bone density in women: the role of androgens, estrogen, and exercise. J Bone Miner Res 3: 673-680, 1988.

13. Burr DB, Robling AG, and Turner CH. Effects of biomechanical stress on bones in animals. Bone 30: 781-786, 2002.

14. Centers for Disease Control and Prevention (CDC). Behavioral Risk Factor Surveillance System Survey Data. Atlanta, GA: U.S. Department of Health and Human Services, Centers for Disease Control and Prevention, 2002.

15. Cheng S, Sipila S, Taaffe DR, Puolakka J, and Suominen H. Change in bone mass distribution induced by hormone replacement therapy and high-impact physical exercise in post-menopausal women. Bone 31: 126-135, 2002.

16. Chilibeck P, Calder A, Sale DG, and Webber C. Reproducibility of dual-energy x-ray absorptiometry. Can Assoc Radiol J 45: 297-302, 1994. 
17. Compston JE. Sex steroids and bone. Physiol Rev 81: 419-447, 2001.

18. Coupland CA, Cliffe SJ, Bassey EJ, Grainge MJ, Hosking DJ, and Chilvers

CE. Habitual physical activity and bone mineral density in postmenopausal women in England. Int J Epidemiol 28: 241-246, 1999.

19. Creighton DL, Morgan AL, Boardley D, and Brolinson PG. Weight-bearing exercise and markers of bone turnover in female athletes. J Appl Physiol 90: 565570,2001

20. Dalsky GP, Stocke KS, Ehsani AA, Slatopolsky E, Lee WC, and Birge SJ, Jr. Weight-bearing exercise training and lumbar bone mineral content in postmenopausal women. Ann Intern Med 108: 824-828, 1988.

21. Davee AM, Rosen CJ, and Adler RA. Exercise patterns and trabecular bone density in college women. J Bone Miner Res 5: 245-250, 1990.

22. Dook JE, James C, Henderson NK, and Price RI. Exercise and bone mineral density in mature female athletes. Med Sci Sports Exerc 29: 291-296, 1997.

23. Ebben WPaRLJ. Electromyographic and Kinetic Analysis of Traditional, Chain, and Elastic Band Squats. Journal or Strength and Conditioning Research 16: 547$550,2002$.

24. Ernst E. Exercise for female osteoporosis. A systematic review of randomised clinical trials. Sports Med 25: 359-368, 1998.

25. Feskanich D, Willett W, and Colditz G. Walking and leisure-time activity and risk of hip fracture in postmenopausal women. Jama 288: 2300-2306, 2002. 
26. Friedlander AL, Genant HK, Sadowsky S, Byl NN, and Gluer CC. A two-year program of aerobics and weight training enhances bone mineral density of young women. J Bone Miner Res 10: 574-585, 1995.

27. Fuchs RK, Bauer JJ, and Snow CM. Jumping improves hip and lumbar spine bone mass in prepubescent children: a randomized controlled trial. J Bone Miner Res 16: 148-156, 2001.

28. Greenspan SL, Maitland LA, Myers ER, Krasnow MB, and Kido TH. Femoral bone loss progresses with age: a longitudinal study in women over age 65 . J Bone Miner Res 9: 1959-1965, 1994.

29. Grove KA and Londeree BR. Bone density in postmenopausal women: high impact vs low impact exercise. Med Sci Sports Exerc 24: 1190-1194, 1992.

30. Gutin B and Kasper MJ. Can vigorous exercise play a role in osteoporosis prevention? A review. Osteoporos Int 2: 55-69, 1992.

31. Hawkins SA, Schroeder ET, Dreyer HC, Underwood S, and Wiswell RA. Fiveyear maintenance of bone mineral density in women master runners. Med Sci Sports Exerc 35: 137-144, 2003.

32. Heinonen A, Kannus P, Sievanen H, Oja P, Pasanen M, Rinne M, Uusi-Rasi K, and Vuori I. Randomised controlled trial of effect of high-impact exercise on selected risk factors for osteoporotic fractures. Lancet 348: 1343-1347, 1996.

33. Heinonen A, Kannus P, Sievanen H, Pasanen M, Oja P, and Vuori I. Good maintenance of high-impact activity-induced bone gain by voluntary, unsupervised 
exercises: An 8-month follow-up of a randomized controlled trial. J Bone Miner Res 14: 125-128, 1999.

34. Heinrich CH, Going SB, Pamenter RW, Perry CD, Boyden TW, and Lohman TG. Bone mineral content of cyclically menstruating female resistance and endurance trained athletes. Med Sci Sports Exerc 22: 558-563, 1990.

35. Holbrook TL, Barrett-Connor E, Klauber M, and Sartoris D. A populationbased comparison of quantitative dual-energy X-ray absorptiometry with dualphoton absorptiometry of the spine and hip. Calcif Tissue Int 49: 305-307, 1991.

36. Honda A, Umemura Y, and Nagasawa S. Effect of high-impact and lowrepetition training on bones in ovariectomized rats. J Bone Miner Res 16: 1688$1693,2001$.

37. Humphries B, Newton RU, Bronks R, Marshall S, McBride J, TriplettMcBride T, Hakkinen K, Kraemer WJ, and Humphries N. Effect of exercise intensity on bone density, strength, and calcium turnover in older women. Med Sci Sports Exerc 32: 1043-1050, 2000.

38. Ilich JZ and Kerstetter JE. Nutrition in bone health revisited: a story beyond calcium. J Am Coll Nutr 19: 715-737, 2000.

39. Ito M, Nakamura T, Ikeda S, Tahara Y, Hashmi R, Tsurusaki K, Uetani M, and Hayashi K. Effects of lifetime volleyball exercise on bone mineral densities in lumbar spine, calcaneus and tibia for pre-, peri- and postmenopausal women.

Osteoporos Int 12: 104-111, 2001. 
40. Judex S, Donahue LR, and Rubin C. Genetic predisposition to low bone mass is paralleled by an enhanced sensitivity to signals anabolic to the skeleton. Faseb $J 16$ : U287-U303, 2002.

41. Kelley GA, Kelley KS, and Tran ZV. Resistance training and bone mineral density in women: a meta-analysis of controlled trials. Am J Phys Med Rehabil 80: 65-77, 2001.

42. Kemper HC, Twisk JW, van Mechelen W, Post GB, Roos JC, and Lips P. A fifteen-year longitudinal study in young adults on the relation of physical activity and fitness with the development of the bone mass: The Amsterdam Growth And Health Longitudinal Study. Bone 27: 847-853, 2000.

43. Khan K, McKay HA, Haapasalo H, Bennell KL, Forwood MR, Kannus P, and Wark JD. Does childhood and adolescence provide a unique opportunity for exercise to strengthen the skeleton? J Sci Med Sport 3: 150-164, 2000.

44. Kirk S, Sharp CF, Elbaum N, Endres DB, Simons SM, Mohler JG, and Rude RK. Effect of long-distance running on bone mass in women. J Bone Miner Res 4: $515-522,1989$.

45. Kodama Y, Umemura Y, Nagasawa S, Beamer WG, Donahue LR, Rosen CR, Baylink DJ, and Farley JR. Exercise and mechanical loading increase periosteal bone formation and whole bone strength in $\mathrm{C} 57 \mathrm{BL} / 6 \mathrm{~J}$ mice but not in $\mathrm{C} 3 \mathrm{H} / \mathrm{Hej}$ mice. Calcif Tissue Int 66: 298-306, 2000. 
46. Kohrt WM, Ehsani AA, and Birge SJ, Jr. Effects of exercise involving predominantly either joint-reaction or ground-reaction forces on bone mineral density in older women. J Bone Miner Res 12: 1253-1261, 1997.

47. Kroger H, Tuppurainen M, Honkanen R, Alhava E, and Saarikoski S. Bone mineral density and risk factors for osteoporosis--a population-based study of 1600 perimenopausal women. Calcif Tissue Int 55: 1-7, 1994.

48. Lanyon LE. Using functional loading to influence bone mass and architecture: objectives, mechanisms, and relationship with estrogen of the mechanically adaptive process in bone. Bone 18: 37S-43S, 1996.

49. MacKelvie K. Physical Activity and Bone in Childhood and Adolescence. In: Physical Activity and Bone Health, edited by Khan K, McKay, H., Kannus, P., Bailey, D., Wark, J., Bennell, K. Champaign, IL: Human Kinetics, 2001, p. 111128.

50. Maddalozzo GF and Snow CM. High intensity resistance training: effects on bone in older men and women. Calcif Tissue Int 66: 399-404, 2000.

51. Marcus R. Role of exercise in preventing and treating osteoporosis. Rheum Dis Clin North Am 27: 131-141, 2001.

\section{Mares-Perlman JA, Klein BE, Klein R, Ritter LL, Fisher MR, and}

Freudenheim JL. A diet history questionnaire ranks nutrient intakes in middleaged and older men and women similarly to multiple food records. $J$ Nutr 123: 489$501,1993$. 
53. Mazess RB and Barden HS. Bone density in premenopausal women: effects of age, dietary intake, physical activity, smoking, and birth-control pills. Am J Clin Nutr 53: 132-142, 1991.

54. Michel BA, Lane NE, Bjorkengren A, Bloch DA, and Fries JF. Impact of running on lumbar bone density: a 5-year longitudinal study. J Rheumatol 19: 1759$1763,1992$.

55. New SA. Exercise, bone and nutrition. Proc Nutr Soc 60: 265-274, 2001.

56. Nguyen TV, Kelly PJ, Sambrook PN, Gilbert C, Pocock NA, and Eisman JA. Lifestyle factors and bone density in the elderly: implications for osteoporosis prevention. J Bone Miner Res 9: 1339-1346, 1994.

57. Nguyen TV, Sambrook PN, and Eisman JA. Bone loss, physical activity, and weight change in elderly women: the Dubbo Osteoporosis Epidemiology Study. $J$ Bone Miner Res 13: 1458-1467, 1998.

58. Nichols DL, Sanborn CF, Bonnick SL, Gench B, and DiMarco N. Relationship of regional body composition to bone mineral density in college females. Med Sci Sports Exerc 27: 178-182, 1995.

59. Nichols DL, Sanborn CF, and Love AM. Resistance training and bone mineral density in adolescent females. J Pediatr 139: 494-500, 2001.

60. Nieves JW, Golden AL, Siris E, Kelsey JL, and Lindsay R. Teenage and current calcium intake are related to bone mineral density of the hip and forearm in women aged 30-39 years. Am J Epidemiol 141: 342-351, 1995. 
61. Nordin MaF, Victor. Biomechanics of Bone. In: Basic Biomechanics of the Musculoskeletal System (Second edition ed.), edited by Frankel MNaV. Philadelphia, PA: Williams \& Wilkins, a Waverly Company, 1989, p. 6-9.

62. Orwoll ES, Bauer DC, Vogt TM, and Fox KM. Axial bone mass in older women. Study of Osteoporotic Fractures Research Group. Ann Intern Med 124: 187-196, 1996.

63. Peterson SE, Peterson MD, Raymond G, Gilligan C, Checovich MM, and Smith EL. Muscular strength and bone density with weight training in middle-aged women. Med Sci Sports Exerc 23: 499-504, 1991.

64. Pettersson U, Nordstrom P, Alfredson H, Henriksson-Larsen K, and Lorentzon R. Effect of high impact activity on bone mass and size in adolescent females: A comparative study between two different types of sports. Calcif Tissue Int 67: 207-214, 2000.

65. Pocock NA, Eisman JA, Yeates MG, Sambrook PN, and Eberl S. Physical fitness is a major determinant of femoral neck and lumbar spine bone mineral density. J Clin Invest 78: 618-621, 1986.

66. Reid IR, Ames R, Evans MC, Sharpe S, Gamble G, France JT, Lim TM, and Cundy TF. Determinants of total body and regional bone mineral density in normal postmenopausal women--a key role for fat mass. J Clin Endocrinol Metab 75: 45$51,1992$.

67. Riggs BL and Melton LJ, 3rd. The worldwide problem of osteoporosis: insights afforded by epidemiology. Bone 17: 505S-511S, 1995. 
68. Robling AG, Burr DB, and Turner CH. Partitioning a daily mechanical stimulus into discrete loading bouts improves the osteogenic response to loading. J Bone Miner Res 15: 1596-1602, 2000.

69. Robling AG, Burr DB, and Turner CH. Recovery periods restore mechanosensitivity to dynamically loaded bone. J Exp Biol 204: 3389-3399, 2001.

70. Robling AG, Hinant FM, Burr DB, and Turner CH. Improved bone structure and strength after long-term mechanical loading is greatest if loading is separated into short bouts. J Bone Miner Res 17: 1545-1554, 2002.

71. Robling AG, Hinant FM, Burr DB, and Turner CH. Shorter, more frequent mechanical loading sessions enhance bone mass. Med Sci Sports Exerc 34: 196$202,2002$.

72. Shimegi S, Yanagita M, Okano H, Yamada M, Fukui H, Fukumura Y, Ibuki Y, and Kojima I. Physical exercise increases bone mineral density in postmenopausal women. Endocr J 41: 49-56, 1994.

73. Sievanen H, Kannus P, Nieminen V, Heinonen A, Oja P, and Vuori I. Estimation of various mechanical characteristics of human bones using dual energy X-ray absorptiometry: methodology and precision. Bone 18: 17S-27S, 1996.

74. Sievanen H, Kannus P, Oja P, and Vuori I. Dual energy X-ray absorptiometry is also an accurate and precise method to measure the dimensions of human long bones. Calcif Tissue Int 54: 101-105, 1994. 
75. Sievanen H, Oja P, and Vuori I. Precision of dual-energy X-ray absorptiometry in determining bone mineral density and content of various skeletal sites. $J$ Nucl Med 33: 1137-1142, 1992.

76. Sinaki M, Fitzpatrick LA, Ritchie CK, Montesano A, and Wahner HW. Sitespecificity of bone mineral density and muscle strength in women: job-related physical activity. Am J Phys Med Rehabil 77: 470-476, 1998.

77. Snow CM, Shaw JM, Winters KM, and Witzke KA. Long-term exercise using weighted vests prevents hip bone loss in postmenopausal women. J Gerontol A Biol Sci Med Sci 55: M489-491, 2000.

78. Snow-Harter C and Marcus R. Exercise, bone mineral density, and osteoporosis. Exerc Sport Sci Rev 19: 351-388, 1991.

79. Sugiyama T, Yamaguchi A, and Kawai S. Effects of skeletal loading on bone mass and compensation mechanism in bone: a new insight into the "mechanostat" theory. J Bone Miner Metab 20: 196-200, 2002.

80. Suleiman S, Nelson M, Li F, Buxton-Thomas M, and Moniz C. Effect of calcium intake and physical activity level on bone mass and turnover in healthy, white, postmenopausal women. Am J Clin Nutr 66: 937-943, 1997.

81. Teegarden D, Proulx WR, Kern M, Sedlock D, Weaver CM, Johnston CC, and Lyle RM. Previous physical activity relates to bone mineral measures in young women. Med Sci Sports Exerc 28: 105-113, 1996.

82. Tudor-Locke $\mathbf{C}$ and McColl RS. Factors related to variation in premenopausal bone mineral status: a health promotion approach. Osteoporos Int 11: 1-24, 2000. 
83. Turner CH. Functional determinants of bone structure: beyond Wolff's law of bone transformation. Bone 13: 403-409, 1992.

84. Turner CH. Three rules for bone adaptation to mechanical stimuli. Bone 23: 399407, 1998.

85. Turner CH, Forwood MR, and Otter MW. Mechanotransduction in bone: do bone cells act as sensors of fluid flow? Faseb J 8: 875-878, 1994.

86. Turner CH, Owan I, and Takano Y. Mechanotransduction in bone: role of strain rate. Am J Physiol 269: E438-442, 1995.

87. Turner CH and Robling AG. Designing exercise regimens to increase bone strength. Exerc Sport Sci Rev 31: 45-50, 2003.

88. Umemura Y, Ishiko T, Yamauchi T, Kurono M, and Mashiko S. Five jumps per day increase bone mass and breaking force in rats. J Bone Miner Res 12: 1480$1485,1997$.

89. Umemura Y, Sogo N, and Honda A. Effects of intervals between jumps or bouts on osteogenic response to loading. J Appl Physiol 93: 1345-1348, 2002.

90. Uusi-Rasi K, Sievanen H, Pasanen M, Oja P, and Vuori I. Association of physical activity and calcium intake with the maintenance of bone mass in premenopausal women. Osteoporos Int 13: 211-217, 2002.

91. Uusi-Rasi K, Sievanen H, Pasanen M, Oja P, and Vuori I. Maintenance of body weight, physical activity and calcium intake helps preserve bone mass in elderly women. Osteoporos Int 12: 373-379, 2001. 
92. Uusi-Rasi K, Sievanen H, Vuori I, Pasanen M, Heinonen A, and Oja P. Associations of physical activity and calcium intake with bone mass and size in healthy women at different ages. J Bone Miner Res 13: 133-142, 1998.

93. Wallace BA and Cumming RG. Systematic review of randomized trials of the effect of exercise on bone mass in pre- and postmenopausal women. Calcif Tissue Int 67: 10-18, 2000.

94. Wang MC, Crawford PB, Hudes M, Van Loan M, Siemering K, and Bachrach LK. Diet in midpuberty and sedentary activity in prepuberty predict peak bone mass. Am J Clin Nutr 77: 495-503, 2003.

95. Ward JA, Lord SR, Williams P, Anstey K, and Zivanovic E. Physiologic, health and lifestyle factors associated with femoral neck bone density in older women. Bone 16: 373S-378S, 1995.

96. Weaver CM, Teegarden D, Lyle RM, McCabe GP, McCabe LD, Proulx W, Kern M, Sedlock D, Anderson DD, Hillberry BM, Peacock M, and Johnston CC. Impact of exercise on bone health and contraindication of oral contraceptive use in young women. Med Sci Sports Exerc 33: 873-880, 2001.

97. Welsh L and Rutherford OM. Hip bone mineral density is improved by highimpact aerobic exercise in postmenopausal women and men over 50 years. Eur $J$ Appl Physiol Occup Physiol 74: 511-517, 1996.

98. Witzke KA and Snow CM. Effects of plyometric jump training on bone mass in adolescent girls. Med Sci Sports Exerc 32: 1051-1057, 2000. 
99. Wolff I, van Croonenborg JJ, Kemper HC, Kostense PJ, and Twisk JW. The effect of exercise training programs on bone mass: a meta-analysis of published controlled trials in pre- and postmenopausal women. Osteoporos Int 9: 1-12, 1999.

100. Wolman RL, Faulmann L, Clark P, Hesp R, and Harries MG. Different training patterns and bone mineral density of the femoral shaft in elite, female athletes. Ann Rheum Dis 50: 487-489, 1991.

101. Wood R. Calcium and Phosphorus. In: Biochemical and Physiological Aspects of Human Nutrition, edited by Stipanuk M. Philadelphia, PA: W.B. Saunders Co., 2000, p. 643-670.

102. Zhang J, Feldblum PJ, and Fortney JA. Moderate physical activity and bone density among perimenopausal women. Am J Public Health 82: 736-738, 1992.

103. Zylstra S, Hopkins A, Erk M, Hreshchyshyn MM, and Anbar M. Effect of physical activity on lumbar spine and femoral neck bone densities. Int J Sports Med 10: 181-186, 1989. 
Appendix A-1

Informed Consent 


\section{Consent To Be a Research Subject (please read carefully)}

\section{Introduction}

This research study is being conducted by Eric Strong, a Ph.D. candidate at BYU, to determine the effects of stretching on hip and spine bone strength in premenopausal women over 16-weeks. You were selected because you did not meet the exclusion criteria.

\section{Procedures}

Data will be collected seven times, once a month, over 24 weeks. During your first appointment, a menstrual cycle and nutrition questionnaire will be completed. During four of your appointments you will be asked to perform the stretches, be weighed, and have a hip and spine bone scan. During your other three appointments you will be weighed and perform stretches. You will also be asked to stretch at home for 24 weeks. All activities, except for the stretches, will be completed at our facility located in the Richards Building at BYU. Parking passes will be provided so you can park near the building.

Bone scans will be performed by a dual-energy x-ray absorptiometer (DEXA). The DEXA assessment will require you to lie on a table while a lens above you moves back and forth scanning your body. Body height measurement and questionnaires will be completed on the first visit. Instructions for the questionnaires will be given prior to the completion of the questionnaire.

Approximately 20 minutes will be required for each of the four appointments that include the bone scans and weight measurement. Only 5 minutes will be required for each of the three appointments that involve jumping and weight measurement and no bone scan. The time required to fill out the two questionnaires will be approximately 40 minutes. Stretching at home will require approximately 10 minutes per day, six days per week.

\section{Risks/Discomforts}

The risks and discomforts associated with the assessments you will receive will be minimal. Stretching may cause some muscle and joint soreness because you are probably not used to stretching.

The bone scans will expose you to a very small amount of x-ray radiation. To put the amount in perspective, every day we are exposed to radiation from the sun, TV, cell phones, etc. Your DEXA bone assessment will expose you to less radiation than you would typically get from a day of snow skiing or during a round trip flight coast-to-coast in an airplane. The DEXA assessment will have an effective radiation dose that is many times less than a dental X-ray. Steps will be taken to minimize these risks. 


\section{Benefits}

You will receive many benefits from participating in this study. You will receive valuable information regarding your current bone strength at the hip and spine as well as a chance to improve hip and spine bone density from stretching. Bone scans for the hip and scan are worth at least $\$ 90$ each time they are assessed.

\section{Confidentiality}

All data from this study will be kept strictly confidential and will only be reported as group data with no identifying information. Data will be coded and stored in a locked office to ensure confidentiality. Personal information will be destroyed following the completion of the research project.

\section{Compensation}

Participants will receive hip and spine bone density scans and analyses along with a stretching program designed to improve bone density.

\section{Participation}

Participation in this research is voluntary. You have the right to refuse to participate and the right to withdraw from this study at any time without penalty. However, you will only receive your test results if you complete all of the study.

\section{Questions about the research}

If you have any questions regarding this research project, you may contact Eric Strong at 422-1845 or jes26@email.byu.edu.

\section{Questions about your Rights as Research Participants}

If you have questions you do not feel comfortable asking the researcher, you may contact Dr. Shane Schulthies, IRB Chair, 422-5490, 120B RB, shane_schulties@byu.edu

I have read, understood, and received a copy of the above consent form and desire of my own free will and volition to participate in this study. 


\section{Consent To Be a Research Subject (please read carefully)}

\section{Introduction}

This research study is being conducted by Eric Strong, a Ph.D. candidate at BYU, to determine the effects of exercise on hip and spine bone strength in premenopausal women over 24-weeks. You were selected because you did not meet the exclusion criteria.

\section{Procedures}

Data will be collected seven times, once a month, over 24 weeks. During your first appointment, a menstrual cycle and nutrition questionnaire will be completed. During four of your appointments you will be asked to jump as high as possible about 10 times, have a hip and spine bone scan, and be weighed. During your other three appointments you will be asked to jump about 10 times and you will be weighed. You will also be asked to do jumping exercises at home for 24 weeks. All activities, except for the jumping exercises, will be completed at our facility located in the Richards Building at BYU. Parking passes will be provided so you can park near the building.

Bone scans will be performed by a dual-energy x-ray absorptiometer (DEXA). The DEXA assessment will require you to lie on a table while a lens above you moves back and forth scanning your body. Leg power will be determined by jumping as high as possible onto a platform that measures force. Body height measurement and questionnaires will be completed on the first visit. Instructions for the questionnaires will be given prior to the completion of the questionnaire.

Approximately 30 minutes will be required for each of the three appointments that include the bone scans, weight measurement, and jumping. Only 15 minutes will be required for each of the two appointments that involve jumping and weight measurement and no bone scan. The time required to fill out the two questionnaires will be approximately 40 minutes. Jumping exercises at home will require approximately 15-20 minutes per day, six days per week.

\section{Risks/Discomforts}

The risks and discomforts associated with the assessments you will receive will be minimal. The jumping assessment may cause some soreness because you are probably not used to jumping. The risks that accompany the jump assessment will be the same as they would be if you participated in basketball or volleyball. When jumping, you could hurt a joint or muscle, or you could fall and injure yourself.

The bone scans will expose you to a very small amount of x-ray radiation. To put the amount in perspective, every day we are exposed to radiation from the sun, TV, cell phones, etc. Your DEXA bone assessment will expose you to less radiation than you would typically get from a day of snow skiing or during a round trip flight coast-to-coast 
in an airplane. The DEXA assessment will have an effective radiation dose that is many times less than a dental X-ray. Steps will be taken to minimize these risks.

\section{Benefits}

You will receive many benefits from participating in this study. You will receive valuable information regarding your current bone strength at the hip and spine as well as a chance to improve hip and spine bone density from exercising. Bone scans for the hip and scan are worth $\$ 90$ each time they are assessed.

\section{Confidentiality}

All data from this study will be kept strictly confidential and will only be reported as group data with no identifying information. Data will be coded and stored in a locked office to ensure confidentiality. Personal information will be destroyed following the completion of the research project.

\section{Compensation}

Participants will receive hip and spine bone density scans and analyses along with an exercise program designed to improve bone density.

\section{Participation}

Participation in this research is voluntary. You have the right to refuse to participate and the right to withdraw from this study at any time without penalty. However, you will only receive your test results if you complete all of the study.

\section{Questions about the research}

If you have any questions regarding this research project, you may contact Eric Strong at 422-1845 or jes26@email.byu.edu.

\section{Questions about your Rights as Research Participants}

If you have questions you do not feel comfortable asking the researcher, you may contact Dr. Shane Schulthies, IRB Chair, 422-5490, 120B RB, shane_schulties@byu.edu

I have read, understood, and received a copy of the above consent form and desire of my own free will and volition to participate in this study.

Research Participant

\section{Date}

Witness 
Appendix A-2

Screening Form 
Age

Between 30 and 45 years

Weight

Height

BMI Not $>25 \mathrm{~kg} / \mathrm{m}^{2}$

YES NO

Smoking

Alcohol consumption

Medical Conditions (Diagnosed within the last 12 months)

Thyroid disorder

Parathyroid disorder

Renal dysfunction

Hepatic dysfunction

Digestive disorder

Arthritis (LE)

Back pain

Heart condition

Malignancy

Do you have an ex. program

If so, Type, Freq., Time, Intensity,

Pregnant Now

Pregnant Last 12 months

Breast Feeding

Lost or Gained greater than 10 pounds within the last six months

Current dieter

Planning to Diet in the next 4 mos.

Irregular menstrual cycles If yes, \# per year?

(less than 10 cycles per year)

Hysterectomy

Without HRT

Start HRT within 6 mos.

Use of medications within 6 mos.

Glucocorticoids Anti-convulsants Thiazide diuretics 
Appendix A-3

Menstrual Cycle Questionnaire 
Which of the following best describes your menstrual periods?
A. I don't have them anymore
B. They're very irregular
C. They're irregular
D. They're fairly regular
E. They're very regular

If you responded 'A' above “I don't have them anymore," briefly explain on the line below why you don't have menstrual periods anymore (e.g. menopause, had a hysterectomy, etc):

Please write the number of menstrual cycles you experienced in the last year

What is the usual number of days from start to start (between cycles)? (22-35 days)

Which of the following best describes your menopausal status?
A. I'm definitely not experiencing any symptoms of menopause and I'm not taking medication to prevent it
B. I'm definitely not experiencing any symptoms of menopause, but I am taking medication to prevent it
C. I'm not sure, but I seem to be starting to have a few symptoms of menopause, but I ' $\mathrm{m}$ still having periods
D. I'm confident that I am having some symptoms of menopause but I'm still having periods
E. I'm confident that I am having symptoms of menopause and my periods have become quite irregular
F. I'm confident that I am having symptoms of menopause and my periods have nearly stopped
G. I have stopped having periods because I have reached menopause
H. None of the above apply to me (Please explain below)
If you answered 'H,' please explain here 
Which of the following best describes your experience with symptoms of menopause like hot flashes, or irregular menstrual periods, or vaginal dryness, etc.?
A. I have not yet had any signs or symptoms of menopause
B. I am just beginning to experience some signs and/or symptoms of menopause
C. I began having symptoms of menopause less than six months ago
D. I began having symptoms of menopause 6-12 months ago
E. I began having symptoms of menopause 1-2 years ago and I'm still having symptoms
F. I began having symptoms of menopause 2-3 years ago and I'm still having symptoms

G. For several months I have had symptoms of menopause, but they now seem to be almost over

H. For several months I have had symptoms of menopause but they have finally stopped

Which of the following is most accurate regarding how long you have been on HRThormone replacement therapy- (usually estrogen), other than for birth control?
A. I have never taken estrogen for hormone replacement therapy
B. I used to be on hormone replacement therapy, but haven't been for over a year
C. I used to be on hormone replacement therapy, but haven't been for less than a year
D. I've been taking estrogen (HRT) regularly for less than six months
E. I've been taking estrogen (HRT) regularly for greater than six month 
Appendix A-4

Exercise Logs 


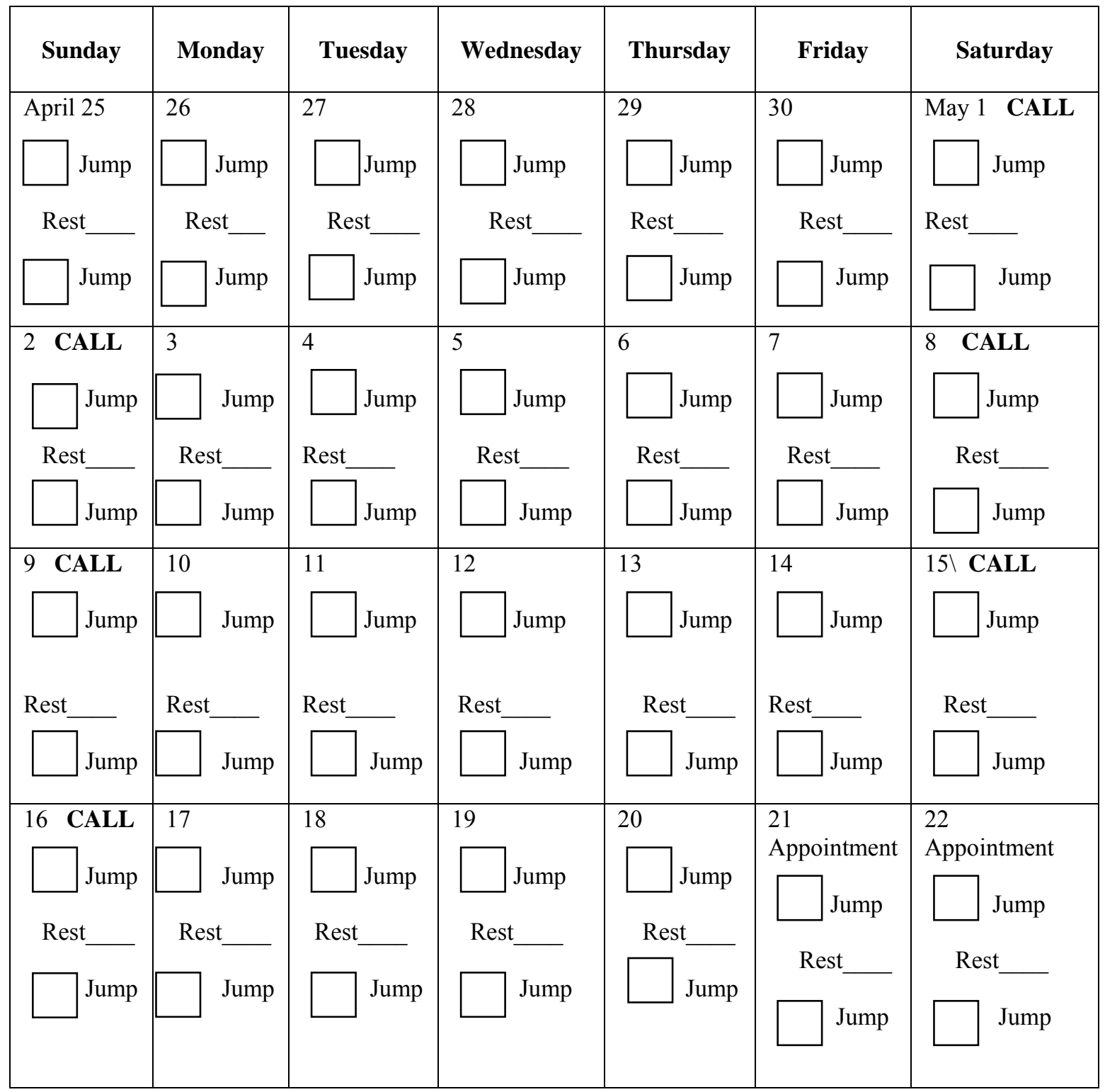

Name 


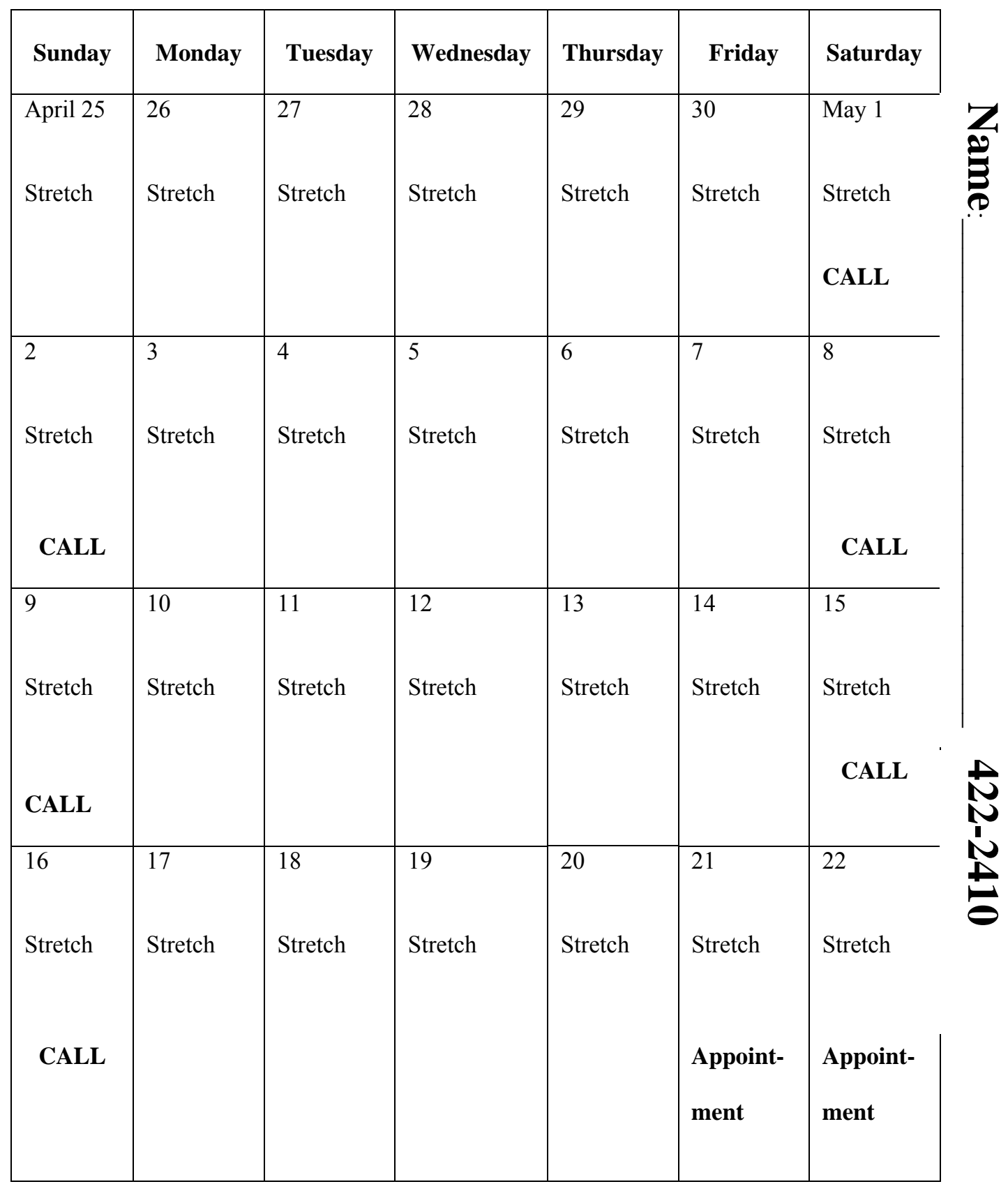

